Article

\title{
Net Absorption and Metabolism of $\beta$-Hydroxy- $\beta$-Methyl Butyrate during Late Gestation in a Pig Model
}

\author{
Liang Hu ${ }^{1,2}$, Niels Bastian Kristensen ${ }^{1,3}$, Uffe Krogh ${ }^{1,4}$ and Peter Kappel Theil ${ }^{1, *}$ \\ 1 Department of Animal Science, Aarhus University, DK-8830 Tjele, Denmark; huliang@anis.au.dk (L.H.); \\ nbk@seges.dk (N.B.K.); uffe.krogh@inrae.fr (U.K.) \\ 2 Institute of Animal Nutrition, Sichuan Agricultural University, No. 211, Huimin Road, Wenjiang District, \\ Chengdu 611130, China \\ 3 Danish Agriculture \& Food Council F.m.b.A. SEGES Agro Food Park 15, DK 8200 Aarhus N, Denmark \\ 4 PEGASE, INRAE, Agrocampus Ouest, 35590 Saint-Gilles, France \\ * Correspondence: Peter.Theil@anis.au.dk; Tel.: +45-8715-7803
}

Received: 29 January 2020; Accepted: 19 February 2020; Published: 21 February 2020

\begin{abstract}
The leucine metabolite, $\beta$-hydroxy- $\beta$-methyl butyrate (HMB), is widely used in human nutrition and animal production as a nutritional supplement. Although the HMB usage during late gestation has been demonstrated to have a positive effect on fetal development, knowledge on net absorption and metabolism of HMB and impact of HMB on branched chain amino acids (BCAAs) metabolism is lacking. To address this, we conducted a study using pigs during the perinatal period as a model organism. Eight-second parity sows were fitted with indwelling catheters in the femoral artery and in the portal, hepatic, femoral, and mesenteric veins. Eight hourly sets of blood samples were taken starting $30 \mathrm{~min}$ before the morning meal on day -10 and day -3 relative to parturition. Four control (CON) sows were fed a standard lactation diet from day -15 and throughout the experiment, and $4 \mathrm{HMB}$ sows were fed the control diet supplemented with $15 \mathrm{mg} \mathrm{Ca}(\mathrm{HMB})_{2} / \mathrm{kg}$ body weight mixed in one third of the morning meal from day -10 until parturition. Blood gases, plasma metabolites, milk compositions, and apparent total tract digestibility of nutrients were measured. Arterial plasma concentrations of $\operatorname{HMB}(p<0.001)$, Cys $(p<0.001)$, and Lys $(p<0.10)$ were increased in HMB supplemented sows, while arterial plasma triglycerides concentration was decreased $(p<0.05)$. The net portal recovery of Ala and Asp were increased in HMB sows $(p<0.05)$. Sows fed HMB had increased hepatic vein flow and net hepatic fluxes of Met, Asn, and Gln $(p<0.05)$. In contrast, the femoral extraction rates of Ala and Ser were decreased by dietary HMB supplementation $(p<0.05)$. Dietary HMB treatment and sampling time relative to feeding had an interaction on arterial concentrations, net portal fluxes, and femoral extraction rates of BCAAs. The net portal recovery of $\mathrm{HMB}$ was $88 \%$, while $14 \%$ of supplemented HMB was excreted through urine and $4 \%$ through feces. Moreover, the gastrointestinal tract metabolized $8 \%$ while the liver metabolized $12 \%$. Finally, $26 \%$ of the daily intake of HMB was secreted via colostrum at the day of farrowing. This study demonstrated that dietary HMB supplementation increased net uptake of amino acids and increased fatty acid oxidation through improving blood flow and insulin sensitivity during the late gestation. Most importantly, oral HMB administration could maintain a stable postprandial absorption and altered metabolism in BCAAs. Net portal flux of HMB at 5.5 to $6.5 \mathrm{~h}$ after feeding approached zero, indicating that HMB ideally should be administrated two or three times, daily.
\end{abstract}

Keywords: amino acids; HMB; leucine metabolites; sow; transition 


\section{Introduction}

$\beta$-hydroxy- $\beta$-methyl butyrate (HMB) is a metabolite of the nutritionally essential branched-chain amino acid (BCAA) leucine [1,2]. Human studies have shown that HMB stimulated muscle growth and increased fat oxidation, and therefore, it is commonly used as a dietary supplement for athletes and for bedridden patients to prevent excessive muscle degeneration [3,4]. Moreover, HMB is also widely applied in animal production as a functional additive over the last decades. For late gestating sows, HMB may increase the colostrum fat content or colostrum yield when supplied at 2 to $2.5 \mathrm{~g} / \mathrm{d}$ during the last 3 to 7 days prior to parturition $[1,5]$.

Leucine catabolism normally involves a transamination process to form $\alpha$-ketoisocaproic acid (KIC), which may be further metabolized into HMB by the enzyme $\alpha$-ketoisocaproate-dioxygenase [2]. In a study with pigs, it was demonstrated that 2 to $10 \%$ of injected leucine was converted to HMB and found in the cytosol [6]. Van Koevering and Nissen also revealed that 34\% of injected HMB was excreted in the urine, but otherwise, the fate of HMB remains unknown [6]. Metabolism of BCAAs are known to interact with each other, and it is generally believed that excessive supply of dietary leucine leads to catabolism of isoleucine and valine $[7,8]$. However, recently excessive supply of isoleucine was shown to reduce growth in post-weaning of pigs [7], whereas, the excessive supply of leucine or valine had no negative effects on growth performance $[9,10]$.

Interest in prenatal nutritional programming has increased in both animal and human research. Moreover, it has also been demonstrated in animal studies that prenatal administration of HMB has a positive impact on postnatal growth and development [11,12]. However, there is an insufficient number of studies concerning the comprehensive distribution of metabolism when HMB is orally administered during late gestation. Owing to the high similarity in anatomy and physiology between pigs and humans, the porcine model has been frequently used to study human nutrition and intermediary metabolism [13]. Therefore, the present study was carried out to (1) study the fate of supplemented HMB and dietary amino acids (AA) to reveal how these metabolites are digested, absorbed, metabolized, secreted and excreted and (2) to study whether HMB supplementation interacts with fates of dietary BCAAs. More specifically, the net portal absorption, net portal recovery and net hepatic metabolism of these metabolites were quantified using multi-catheterized sows and measurements of blood flow using a blood flow marker, as described for growing pigs [14]. In addition, venous-arterial differences across the right hind leg were studied to evaluate extraction rates of HMB by muscle tissue. This experiment was carried out with late gestating sows within the scope of another study [15].

\section{Materials and Methods}

The present experiment complied with Danish law on the humane care and use of experimental animals (The Danish Ministry of Justice, Animal Testing Act [Consolidation Act No. 1306 of 23 November 2007, as amended by Act No. 612 of June 14, 2011]). The protocols used in the current experiment were reviewed and approved by The Danish Animal Experimentation Inspectorate (Ethic Approval Code: 2008/561-1493).

\subsection{Animals, Surgical Procedures and Housing}

Eight-second parity sows (Danish Landrace $\times$ Yorkshire) were used in this study and housed in an intensive care facility at Research Center Foulum, Aarhus University, Denmark. The sows were surgically fitted with indwelling catheters on day $79 \pm 3$ of gestation, including the right femoral artery, the right femoral vein, the portal vein, the portal hepatic vein and the mesenteric vein as described by Kristensen et al. [14]. After surgery catheters were filled with saline containing heparin $(100 \mathrm{IU} / \mathrm{mL}$, Heparin LEO, LEO Pharma A/S, Ballerup, Denmark), benzyl alcohol (0.1\%, Benzylalcohol +99\%, Sigma-Aldrich, St. Louis, MO), and benzylpenicillin (0.2\%, Benzylpenicillin, Panpharma, NordMedica A/S, Copenhagen, Denmark). After surgery, the sows were individually housed in pens $(2.0 \times 2.7 \mathrm{~m})$ with the partly slatted floor $\left(3.0 \mathrm{~m}^{2}\right)$ until end of lactation. Sows were fitted in farrowing railings from 
d -11 and onwards. Room temperature was kept at $20^{\circ} \mathrm{C}$, and the light was turned on from 0700 to $1830 \mathrm{~h}$ and again during the evening meal (2400 to $0030 \mathrm{~h}$ ), except when the sows were farrowing (light turned on $24 \mathrm{~h}$ per $\mathrm{d}$ ). At the end of the experiment (day 28 of lactation), the positions of the catheters were investigated by autopsy to ensure correct placement.

\subsection{Diets and Feeding}

Sows were allocated by body weight (BW) into two groups (4 sows/group). The control sows received a control (CON) diet (Supplementary Table S1) from day -15 and throughout the experiment, and $\mathrm{HMB}$ sows were fed the $\mathrm{CON}$ diet supplemented with $15 \mathrm{mg} \mathrm{Ca}(\mathrm{HMB})_{2} / \mathrm{kg}$ individual $\mathrm{BW}$ mixed in the morning meal from day -10 prior to expected farrowing until parturition, then fed the control diet until day 28. The $\mathrm{Ca}(\mathrm{HMB})_{2}$ was purchased from VWR-Bie and Berntsen (Herlev, Denmark). Feed allowance was in accordance with the recommendations from the Danish Pig Research Center (VSP, Copenhagen, Denmark). Chromic oxide $(0.2 \%)$ was added to the diet as a digestibility marker for measuring the apparent total tract digestibility (ATTD) of nutrients. Feed was supplied three times daily in equal portions at 0800,1600 , and $2400 \mathrm{~h}$, and the feed intake was registered and feed leftovers were recorded and removed daily between 0800 and 1600, except on sampling days, where leftovers from the night meal were removed before the first sampling and leftovers from the morning meal were removed $30 \mathrm{~min}$ after feeding to ensure accurate recording of feed intake directly related to nutrient absorption and metabolism. Feed intake corrected for refusals were recorded on a daily basis. The piglets had no access to creep feed, but both sows and piglets were offered water ad libitum.

\subsection{Sampling and Data Collection}

Blood sampling was taken on $\mathrm{d}-10$ and $\mathrm{d}-3$ relative to expected parturition. On sampling days, continuous infusion of the blood marker, para-amino hippuric acid $(p \mathrm{AH})$, into the mesenteric vein was initiated $1 \mathrm{~h}$ before the first blood sample to obtain a steady state of plasma $p \mathrm{AH}$. The $p \mathrm{AH}$ infusate contained $175 \mathrm{mmol} / \mathrm{L}$ of $p \mathrm{AH}$, was adjusted to $\mathrm{pH} 7.4$, sterile filtered $(0.22 \mu \mathrm{m}$, FPE 214-500, JET Bio-Filtration Products Co., Ltd., Guangzhou, China), and autoclaved. The infusion rate of $p \mathrm{AH}$ was $79.4 \pm 9.3 \mathrm{~mL} /$ hour. Eight sets of blood samples were simultaneously drawn from four catheters (the artery, the portal vein, the hepatic vein and the femoral vein) at hourly intervals from $0.5 \mathrm{~h}$ before to $6.5 \mathrm{~h}$ after feeding. Whole blood was collected for blood gas measurements, while plasma was obtained by centrifuging the blood sample at $1558 \times \mathrm{g}$ at $4{ }^{\circ} \mathrm{C}$ for $12 \mathrm{~min}$ and stored at $-20^{\circ} \mathrm{C}$ until analysis.

The sows were weighed on day -10 , day 2 , and day 28 relative to parturition. All piglets were weighed at birth, and weighed again $24 \mathrm{~h}$ after the birth of the first piglet (BFP) for determination of colostrum yield [16]. Individual piglet weights were registered on day 2 and day 28 after parturition. The piglets did not receive any feed, but had free access to water at all times. At $24 \mathrm{~h}$ after BFP, the litters were equalized to 12 piglets within the experimental groups. Colostrum samples were taken immediately and at $24 \mathrm{~h}$ after birth, and milk was collected after blood sampling on $\mathrm{d} 3$ and 17, after a $0.3 \mathrm{~mL}$ i.v. injection of oxytocin. Spontaneous urine and feces samples were collected on blood sampling days, and aliquots were stored at $-20^{\circ} \mathrm{C}$ for later analysis.

\subsection{Analytical Procedures}

Diets. Dry matter (DM) content was measured by drying to constant weight (approximately $20 \mathrm{~h}$ ) at $103{ }^{\circ} \mathrm{C}$, and ash was analyzed according to the AOAC (2000) method no 942.05. Feed gross energy (GE) was determined using an adiabatic bomb calorimeter (Parr Instrument Company, Moline, Illinois, USA). The crude fiber was analyzed according to the AOAC method (method 978.10, AOAC, 2007). Nitrogen content was measured by the Kjeldahl method (Kjeltec 2400, Foss, Hillerød, Denmark) according to the manufactures instructions and protein was calculated as Nitrogen $\times 6.25$. Fat was extracted with diethyl ether after $\mathrm{HCl}$ hydrolysis according to the Stoldt procedure [17]. Amino acids were quantified after hydrolysis for $23 \mathrm{~h}$ at $110^{\circ} \mathrm{C}$ with or without performic acid oxidation using ion 
exchange chromatography and photometric detection after ninhydrin reaction. Chromic oxide in feed was measured spectrophotometrically after oxidation to chromate.

Whole blood. Hematocrit was immediately determined in arterial blood samples by centrifugation in capillary tubes at $13,000 \times g$ for $6 \mathrm{~min}$ at ambient temperature. Blood $\mathrm{pH}$ and blood gasses were measured using 2 ABL700 Blood Gas Analyzers (Radiometer, Copenhagen, Denmark).

Plasma. For determination of $p \mathrm{AH}$ concentration, plasma was deacetylated before analysis by the method as described by Harvey et al. [18], and determined using a continuous flow analyzer (Seal Analytical Ltd., Burgess Hill, UK). The concentrations of urea were determined by the method described by Marsh et al. [19]. The HMB concentration was analyzed, after ethyl chloroformat derivatization, determined using gas chromatography/mass spectrometry (GC-MS; Finnigan trace GC ultra, CTC analytics). Plasma AA concentration was determined by GC-MS following ethyl chlorformat derivatization. Glucose and lactate were determined using D-glucose oxidase and L-lactate oxidase, respectively (YSI 7100, YSI inc., Yellowstone Springs, OH). Insulin concentration was analyzed using a solid phase, two-site flouroimmunometric assay (AutoDELFIA, Wallac Oy, Turku, Finland). Plasma concentration of volatile fatty acids (VFA) was determined by GC-MS following 2 Chloro-Ethyl-Chloroformat derivatization [20]. Non-esterified fatty acid (NEFA) concentration in plasma was analyzed using commercial kits for Cobas Mira autoanalyzer (Triolab A/S, Brøndby, Denmark).

Urine and feces. Urinary HMB was analyzed as described for plasma. Feces content of nitrogen, $\mathrm{DM}$, ash, chromium oxide, energy, crude fiber, and fat were determined as for the feed. Feces content of HMB was determined using GC-MS.

Colostrum and milk. Milk composition was analyzed for fat, lactose, protein, and DM concentrations by infrared spectroscopy (Milkoscan 4000; Foss MilkoScan, Hillerød, Denmark). The estimated values obtained by the Milkoscan method were corrected using linear models based on reference methods [21]. Concentrations of total AA in freeze-dried milk samples were analyzed using a Biochrom 30+ AA analyzer (Biochrom, Cambridge, UK) by ion exchange chromatography according to the European Union after hydrolysis in $6 \mathrm{M} \mathrm{HCl}$ for $23 \mathrm{~h}$. Sulfur containing AA was separately liberated by oxidation in a hydrogen peroxide and performic acid solution for $16 \mathrm{~h}$ prior to hydrolysis. Milk HMB content was analyzed as described for plasma.

\subsection{Calculations and Statistical Analysis}

Milk intake of the piglets on $\mathrm{d} 3$ and 17 DIM was calculated according to Theil et al. [22]. Sow milk yield was calculated as the sum of milk intake of the piglets within each litter and DIM. Energy and nutrients ATTD were calculated as described by Stein et al. [23]. Plasma flows and blood flows in the portal vein, hepatic vein and the hepatic artery as well as net portal flux (NPF), net hepatic flux (NHF), net portal recovery, and hepatic extraction were calculated according to $\mathrm{Hu}$ et al. [24]. HMB intake $(\mathrm{g} / \mathrm{d})=$ sow $\mathrm{BW} \times 0.015 \times 0.808$, where 0.015 corresponds with $15 \mathrm{mg} / \mathrm{kg}$ BW and 0.808 accounts for $\mathrm{HBM}$ content in $\mathrm{Ca}(\mathrm{HMB})_{2} \cdot 1 \mathrm{H}_{2} \mathrm{O}$ (molar mass $=292.35 \mathrm{~g} / \mathrm{mol}$ ), and when converting into $\mathrm{mmol} / \mathrm{L}$ or $\mathrm{mmol} / \mathrm{h}, 2 \mathrm{~mol}$ of $\mathrm{HMB}$ for each mole of $\mathrm{Ca}(\mathrm{HMB})_{2}$ was taken into account. HMB excretion in urine was calculated from equations below (Equations (1) and (2)), where urine production was calculated according to Feyera et al. [25] as the infusion rate of $p \mathrm{AH}$ divided by urinary $p \mathrm{AH}$ concentration:

HMB excretion in urine $(\mathrm{g} / \mathrm{d})=[\mathrm{HMB}$ concentration in $\mathrm{HMB}$ sow's urine $(\mathrm{mmol} / \mathrm{mL}) \times$ urine volume $(\mathrm{mL} / \mathrm{d})-\mathrm{HMB}$ concentration in CON sow's urine

$(\mathrm{mmol} / \mathrm{mL}) \times$ urine volume $(\mathrm{mL} / \mathrm{d})] \times 118.13(\mathrm{~g} / \mathrm{mol}) \times 0.001(\mathrm{~mol} / \mathrm{mmol})$

HMB excretion in urine relative to intake $(\%)=H M B$ excretion in urine $(\mathrm{g} / \mathrm{d}) /$ HMB intake $(\mathrm{g} / \mathrm{d}) \times 100$ 
HMB content in feces was calculated according to the following equations (Equations (3)-(5)):

Undigested DM $(\mathrm{kg} / \mathrm{d})=$ Average daily feed intake $(\mathrm{kg} / \mathrm{d}) \times[1-\mathrm{DM}$ digestibility

HMB content in feces $(\mathrm{g} / \mathrm{d})=$ Undigested DM $(\mathrm{kg} / \mathrm{d}) \times$ HMB concentration in feces $(\mathrm{mmol} / \mathrm{g}) \times 118.13(\mathrm{~g} / \mathrm{mol}) \times 0.001(\mathrm{~mol} / \mathrm{mmol})$

$\mathrm{HMB}$ in feces relative to intake $(\%)=\mathrm{HMB}$ content in feces $(\mathrm{g} / \mathrm{d}) / \mathrm{HMB}$ intake

$$
(\mathrm{g} / \mathrm{d}) \times 100
$$

HMB metabolized by gastrointestinal tract (GIT) was calculated according to the following equation (Equation (6)):

HMB metabolized by GIT relative to intake $(\%)=100-\mathrm{HMB}$ in feces relative to intake $(\%)$ - Net portal recovery of HMB (\% of intake)

HMB production in colostrum/milk was calculated according to the following equation (Equations (7) and (8)):

HMB secretion through colostrum $/$ milk $(\mathrm{g} / \mathrm{d})=$ yield $(\mathrm{kg} / \mathrm{d}) \times \mathrm{HMB}$ concentration in colostrum/milk $(\mathrm{mmol} / \mathrm{L}) \times 118.13(\mathrm{~g} / \mathrm{mol}) \times 1(\mathrm{~kg} / \mathrm{L}) \times 0.001(\mathrm{~mol} / \mathrm{mmol})$

HMB secretion through colostrum/milk relative to intake $(\%)=\mathrm{HMB}$ secretion through colostrum/milk (g/d)/HMB intake $(\mathrm{g} / \mathrm{d}) \times 100$

HMB metabolism by the liver was calculated according to the following equation (Equations (9) and (10)):

HMB metabolism by liver $(\mathrm{g} / \mathrm{d})=$ Net hepatic flux of $\mathrm{HMB}(\mathrm{mmol} / \mathrm{h}) \times 24 \mathrm{~h} / \mathrm{d} \times$ $118.13(\mathrm{~g} / \mathrm{mol}) \times 0.001(\mathrm{~mol} / \mathrm{mmol})$

HMB metabolism by liver relative to intake $(\%)=$ HMB metabolism by liver $(\mathrm{g} / \mathrm{d}) /$ HMB intake $(\mathrm{g} / \mathrm{d}) \times 100$

The MIXED procedure of SAS (SAS Inst. Inc., Cary, NC) was applied in the statistical analysis using a model, including the fixed effects of treatment (CON, HMB), DIM $(-10,-3)$, time relative to feeding $(-0.5,0.5,1.5,2.5,3.5,4.5,5.5,6.5)$, and all 2-way interactions between fixed effects. The sow was included as a random effect using a compound symmetry covariance structure for accounting for repeated measurements within sows across DIM. To account for repeated measurements within a sampling day, a first order autoregressive covariance structure was applied using a repeated statement. Data were presented as means \pm SEM. A probability value of $p<0.05$ was considered as statistically significant and $p<0.10$ was considered as a tendency.

\section{Results}

\subsection{Feed intake, Sow Performance and Digestibility}

According to the experimental design, HMB sows ingested on average $3.2 \mathrm{~g} / \mathrm{d}$ of HMB, whereas, CON sows did not ingest any HMB from d -10 up until farrowing $(p<0.001$, Table 1$)$. Feed intake, sow BW change, ATTD, and litter performance were not affected by HMB supplementation $(p>0.05)$. 
Table 1. Feed and water intake of sows and performance of sows and piglets during transition and lactation, and apparent total tract digestibility of sow diets.

\begin{tabular}{lcccc}
\hline & CON & HMB & SEM & $p$-Values \\
\hline Sows & & & & \\
HMB intake from d -10 to farrowing, g/d & 0.0 & 3.2 & 0.03 & $<0.001$ \\
Overall feed intake, kg/d & 3.80 & 3.85 & 0.37 & 0.92 \\
Water intake, L/d & 26.0 & 24.8 & 6.9 & 0.89 \\
Sow BW at d -10, kg & 265 & 259 & 7.3 & 0.58 \\
Sow BW loss from d -10 to 2, kg & 20.3 & 15.0 & 2.9 & 0.23 \\
Sow BW loss from d 2 to 28, kg & 21.0 & 19.0 & 5.8 & 0.81 \\
\hline ATTD, \% & & & & \\
DM & 83.5 & 82.2 & 1.0 & 0.28 \\
GE & 83.0 & 81.6 & 1.1 & 0.31 \\
Nitrogen & 84.2 & 83.5 & 1.3 & 0.63 \\
Fat & 62.7 & 58.4 & 2.2 & 0.13 \\
Crude Fiber & 68.2 & 65.9 & 2.4 & 0.45 \\
\hline Piglets & & & & \\
Mean Piglet BW at birth, kg & 1.24 & 1.20 & 0.04 & 0.45 \\
Litter gain, kg/d & 2.38 & 2.36 & 0.17 & 0.92 \\
Litter size at birth & 18.8 & 15.5 & 1.8 & 0.25 \\
Litter size at d 2 (After cross-foster) & 12.0 & 12.3 & 0.2 & 0.29 \\
Litter size at d 28 & 11.7 & 11.0 & 0.7 & 0.56 \\
\hline
\end{tabular}

$\mathrm{CON}=$ control; $\mathrm{HMB}=\beta$-hydroxy- $\beta$-methyl butyrate; $\mathrm{BW}=$ body weight; $\mathrm{ATTD}=$ apparent total tract digestibility; $\mathrm{DM}=$ dry matter; $\mathrm{GE}=$ gross energy; $\mathrm{SEM}=$ standard error of the mean.

\subsection{Yield and Composition of Colostrum and Milk}

The yield and composition in both colostrum and milk were not affected by HMB supplementation $(p>0.05$, Figure 1), except that HMB concentration of colostrum was higher for HMB sows than for CON sows ( $p=0.04$, Figure 1$)$. Moreover, HMB supplementation increased the milk concentrations of Met $(p=0.047)$ and tended to increase the milk concentrations of Glu $(p=0.07$, Table 2$)$. All milk amino acid concentrations were affected by DIM $(p<0.01)$, but no interaction between treatment and DIM was observed $(p>0.05)$.
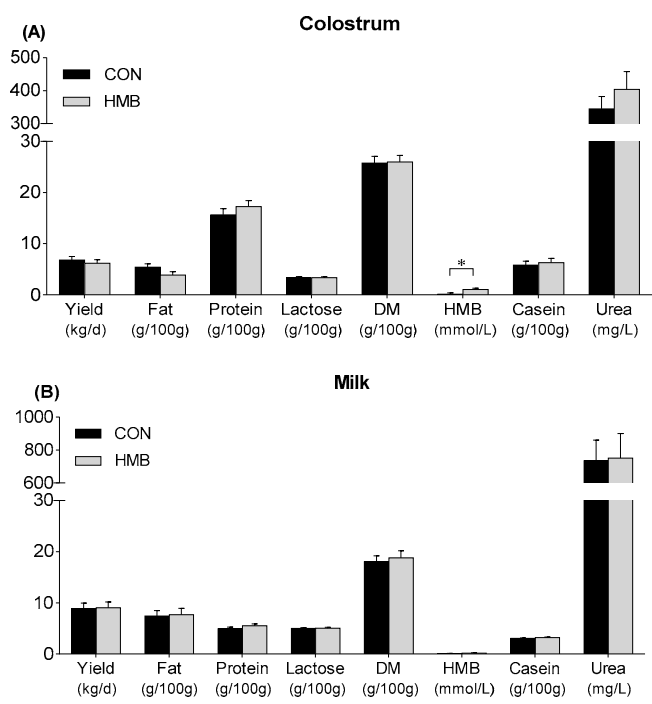

Figure 1. Milk yield and milk composition of colostrum (A) and milk (B) in control (CON) and HMB-supplemented (HMB) sows. Data are expressed as the mean \pm SEM. Sows were regarded as the experimental units, $n=4$ for each group. The differences between groups were indicated by an asterisk $(p<0.05) . \mathrm{CON}=$ control; $\mathrm{HMB}=\beta$-hydroxy- $\beta$-methyl butyrate; $\mathrm{DM}=$ dry matter. 
Table 2. Milk amino acids compositions of control (CON) and HMB-supplemented (HMB) sows during early and peak lactation.

\begin{tabular}{|c|c|c|c|c|c|c|c|c|c|}
\hline \multirow{2}{*}{ Item } & \multicolumn{2}{|c|}{ Treatment } & \multirow{2}{*}{ SEM } & \multicolumn{2}{|c|}{ DIM } & \multirow{2}{*}{ SEM } & \multicolumn{3}{|c|}{$p$-Values } \\
\hline & $\mathrm{CON}$ & НМВ & & 3 & 17 & & Trt & DIM & Trt $\times$ DIM \\
\hline \multicolumn{10}{|c|}{ Essential AA, g/kg milk } \\
\hline Lysine & 3.65 & 3.86 & 0.12 & 4.20 & 3.31 & 0.11 & 0.20 & $<0.001$ & 0.80 \\
\hline Methionine & 0.93 & 1.01 & 0.03 & 1.07 & 0.87 & 0.02 & 0.047 & $<0.001$ & 0.75 \\
\hline Threonine & 2.06 & 2.17 & 0.08 & 2.41 & 1.83 & 0.07 & 0.31 & $<0.001$ & 0.71 \\
\hline Isoleucine & 2.12 & 2.24 & 0.06 & 2.45 & 1.91 & 0.06 & 0.17 & $<0.001$ & 0.72 \\
\hline Leucine & 4.19 & 4.43 & 0.13 & 4.85 & 3.78 & 0.12 & 0.19 & $<0.001$ & 0.70 \\
\hline Valine & 2.74 & 2.89 & 0.09 & 3.18 & 2.45 & 0.09 & 0.24 & $<0.001$ & 0.68 \\
\hline Histidine & 1.33 & 1.41 & 0.04 & 1.54 & 1.20 & 0.04 & 0.17 & $<0.001$ & 0.86 \\
\hline Phenylalanine & 1.98 & 2.07 & 0.07 & 2.27 & 1.77 & 0.06 & 0.32 & $<0.001$ & 0.62 \\
\hline \multicolumn{10}{|c|}{ Nonessential AA, g/kg milk } \\
\hline Alanine & 1.80 & 1.89 & 0.06 & 2.12 & 1.57 & 0.06 & 0.26 & $<0.001$ & 0.60 \\
\hline Aspartate & 4.11 & 4.39 & 0.12 & 4.77 & 3.73 & 0.12 & 0.12 & $<0.001$ & 0.72 \\
\hline Cysteine & 0.72 & 0.74 & 0.03 & 0.84 & 0.62 & 0.03 & 0.63 & $<0.001$ & 0.31 \\
\hline Glutamate & 9.80 & 10.55 & 0.28 & 11.00 & 9.36 & 0.26 & 0.07 & 0.002 & 0.99 \\
\hline Glycine & 1.60 & 1.71 & 0.05 & 1.81 & 1.50 & 0.04 & 0.12 & $<0.001$ & 0.94 \\
\hline Proline & 5.32 & 5.78 & 0.17 & 5.92 & 5.18 & 0.16 & 0.06 & 0.008 & 0.90 \\
\hline Serine & 2.68 & 2.88 & 0.10 & 3.10 & 2.47 & 0.09 & 0.16 & $<0.001$ & 0.85 \\
\hline Arginine & 2.37 & 2.52 & 0.08 & 2.76 & 2.14 & 0.08 & 0.18 & $<0.001$ & 0.76 \\
\hline
\end{tabular}

$\mathrm{CON}=$ control; HMB = $\beta$-hydroxy- $\beta$-methyl butyrate; Trt = treatment; DIM = days in milk; SEM = standard error of the mean; $\mathrm{AA}=$ amino acid.

\subsection{Arterial Variables}

Sows fed HMB had increased arterial plasma concentrations of HMB $(p<0.001$, Table 3$)$ and Cys $(p<0.001)$, and had a tendency to increase Lys concentration $(p=0.09)$ compared to CON sows, whereas, HMB sows had lower arterial plasma concentration of TG $(p=0.046)$ compared to CON sows. The arterial concentrations of $\mathrm{CO}_{2}$, insulin, Leu, Val, Asn, Tyr, Gln, acetate and propionate at DIM -3 were lower than that at DIM -10 $(p<0.05)$. Interaction between treatment and DIM was observed for arterial hematocrit $(p=0.004), \mathrm{O}_{2}(p=0.005)$, Ile $(p=0.03)$, Leu $(p=0.01)$, Asn $(p=0.02)$, and Ser $(p=0.02)$. All measured arterial concentrations of metabolites, except propionate and butyrate, were affected by ST $(p<0.05)$. Interactions between treatment and ST were observed for arterial HMB $(p<0.001)$, TG $(p<0.10)$, insulin $(p<0.001)$, glucose $(p<0.001)$, lactate $(p=0.002)$, and Lys $(p=0.003)$ are illustrated in Figure 2. Interactions between treatment and ST for arterial hematocrit $(p=0.04)$, Thr $(p=0.008)$, Trp $(p=0.009)$, Ile $(p=0.006)$, Leu $(p=0.02)$, Val $(p=0.006)$, Phe $(p=0.003)$, Ala $(p<0.001)$, Asn $(p<0.001), \operatorname{Asp}(p=0.02), \operatorname{Ser}(p=0.003), \operatorname{Tyr}(p=0.009), \operatorname{Gln}(p=0.04)$, and butyrate $(p=0.05)$ are shown in Table 3. 
Table 3. The arterial concentration of blood gases, hematocrit, and plasma metabolites of control (CON) and HMB-supplemented (HMB) sows during late gestation.

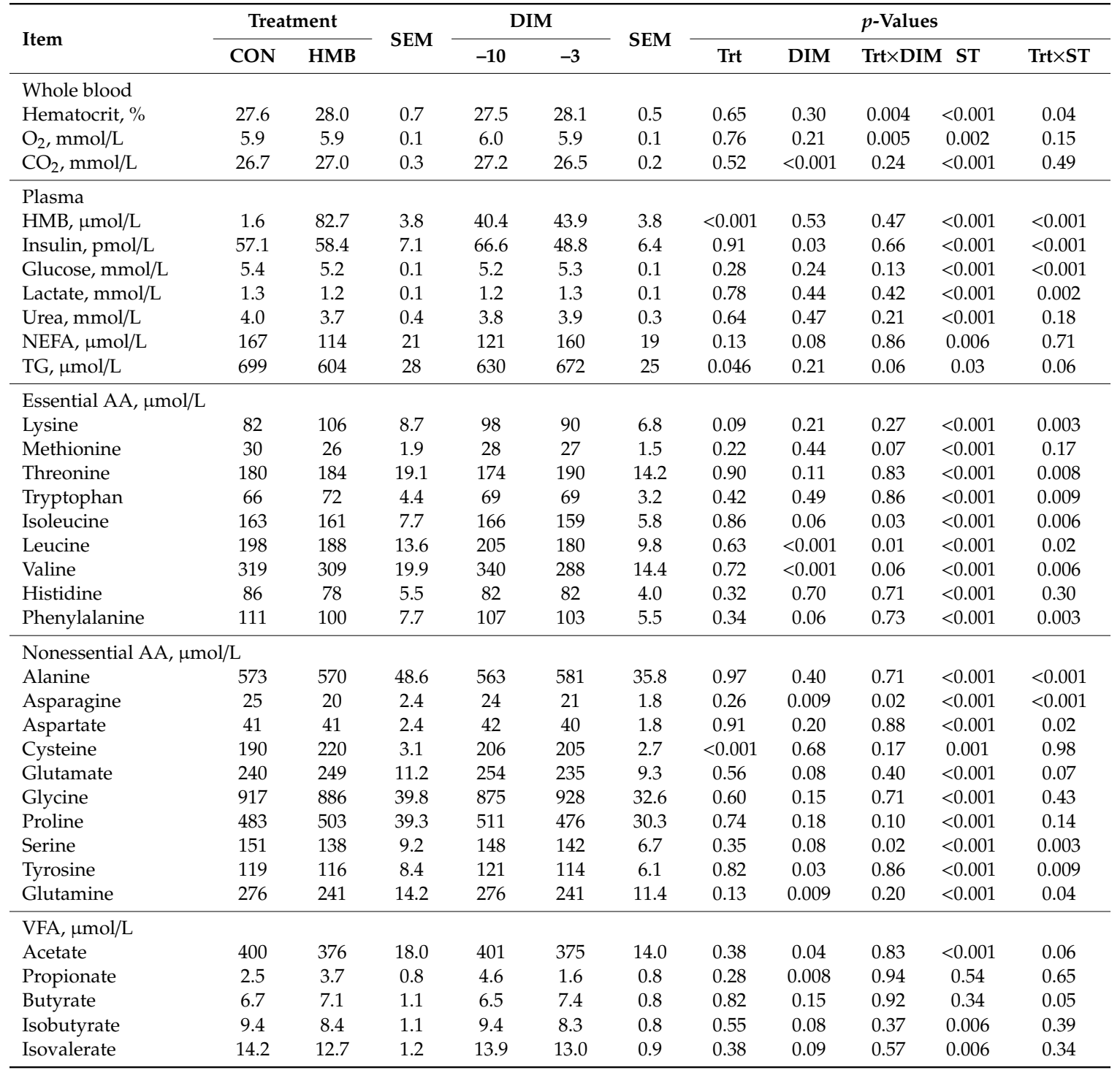

$\mathrm{CON}=$ control $; \mathrm{HMB}=\beta$-hydroxy- $\beta$-methyl butyrate; $\mathrm{Trt}=$ treatment; $\mathrm{DIM}=$ days in milk; $\mathrm{ST}$ = sampling time; $\mathrm{NEFA}=$ non-esterified fatty acids; TG = triglycerides; VFA = volatile fatty acid; SEM = standard error of the mean;

$\mathrm{AA}=$ amino acid 

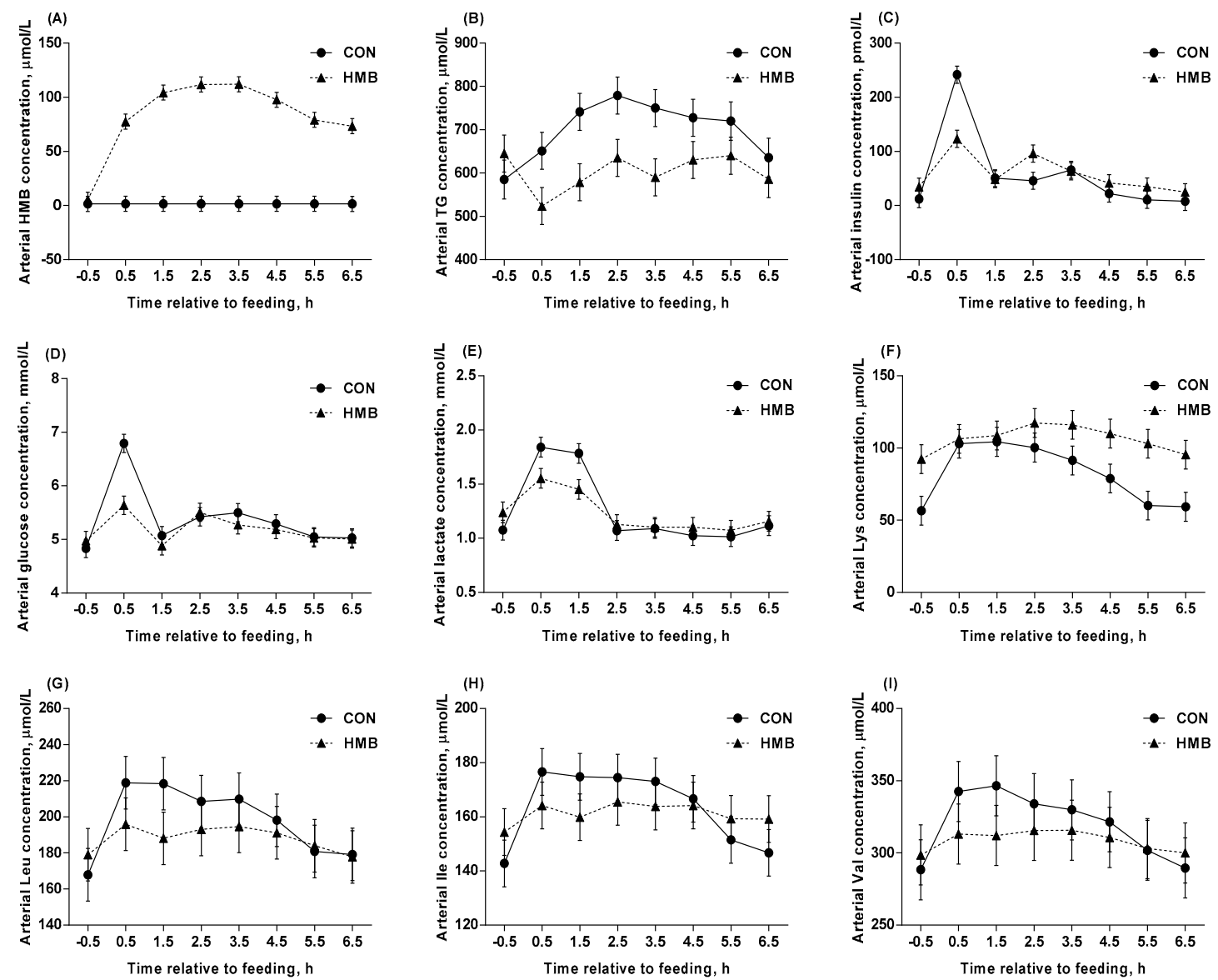

Figure 2. Arterial concentrations of $\operatorname{HMB}(\mathbf{A}), \mathrm{TG}(\mathbf{B})$, insulin (C), glucose (D), lactate (E), Lys (F), Leu $(\mathbf{G})$, Ile $(\mathbf{H})$, and Val (I) in control $(\mathrm{CON})$ and HMB-supplemented (HMB) sows during late gestation. Data are expressed as the mean \pm SEM. Sows were regarded as the experimental units, $n=4$ for each group. Interaction of treatment and sampling time after feeding were observed for arterial concentration of $\mathrm{HMB}(p<0.001)$, TG $(p=0.06)$, insulin $(p<0.001)$, glucose $(p<0.001)$, lactate $(p=0.002)$, Lys $(p=0.003)$, Leu $(p=0.02)$, Ile $(p=0.006)$, and Val $(p=0.006)$, respectively. CON = control; $\mathrm{HMB}=\beta$-hydroxy- $\beta$-methyl butyrate; $\mathrm{TG}=$ triglycerides.

\subsection{Blood Flow, Net Portal Flux, and Net Portal Recovery}

Dietary supplementation of HMB increased the net portal fluxes of HMB $(p<0.001$, Table 4$)$ and Cys $(p=0.005)$, and tended to increase the net portal flux of NEFA $(p=0.09)$, while it tended to decrease the net portal flux of acetate $(p=0.09)$. The net portal fluxes of insulin, lactate, and Ala at DIM -3 were lower than that at DIM -10 $(p<0.05)$. Interaction between treatment and DIM was observed for portal flow, net portal fluxes of insulin, Met and Asn $(p<0.05)$. All measured net portal fluxes, except for urea, NEFA, TG and VFA, were affected by ST $(p<0.05)$. Interactions between treatment and ST was observed for HMB, glucose, insulin, Lys, Leu, Ile, Val, portal flow, and net portal flux of $\mathrm{O}_{2}$ are illustrated in Figure 3. Interaction between treatment and ST was observed in most measured net portal fluxes ( $p<0.05$, Table 4 and Figure 3), except for $\mathrm{CO}_{2}$, lactate, urea, NEFA, TG, Asp, Glu, Gly, Gln and VFA. Relative to feeding, the net portal fluxes of most AA peaked $2.5 \mathrm{~h}$ after feeding in sows fed with $\mathrm{HMB}$, while they peaked $1.5 \mathrm{~h}$ after feeding in CON sows (Figure 3). Dietary supplementation of HMB increased the net portal recovery of Ala $(p=0.04$, Table 5$)$ and Asp $(p=0.003)$. All net portal recovery of AA did not differ across DIM $(p>0.05)$. The net portal recovery of HMB in sows fed HMB was $88 \%$. 
Table 4. Blood and plasma flow, and net portal fluxes of gases and metabolites in control (CON) and HMB-supplemented (HMB) sows during late gestation.

\begin{tabular}{|c|c|c|c|c|c|c|c|c|c|c|c|}
\hline \multirow{2}{*}{ Item } & \multicolumn{2}{|c|}{ Treatment } & \multirow{2}{*}{ SEM } & \multicolumn{2}{|c|}{ DIM } & \multirow{2}{*}{ SEM } & \multicolumn{5}{|c|}{$p$-Values } \\
\hline & CON & HМB & & -10 & -3 & & Trt & DIM & Trt $\times$ DIM & I ST & Trt $\times S T$ \\
\hline \multicolumn{12}{|l|}{ Whole blood } \\
\hline Portal flow, L/h & 161 & 170 & 10 & 166 & 165 & 7 & 0.52 & 0.69 & 0.01 & $<0.001$ & 0.03 \\
\hline $\mathrm{O}_{2}, \mathrm{mmol} / \mathrm{h}$ & -441 & -458 & 13 & -457 & -442 & 12 & 0.42 & 0.30 & 0.21 & 0.001 & 0.04 \\
\hline $\mathrm{CO}_{2}, \mathrm{mmol} / \mathrm{h}$ & 531 & 558 & 22 & 557 & 532 & 22 & 0.40 & 0.36 & 0.10 & 0.09 & 0.29 \\
\hline \multicolumn{12}{|l|}{ Plasma } \\
\hline $\mathrm{HMB}, \mathrm{mmol} / \mathrm{h}$ & 0.0 & 2.4 & 0.1 & 1.2 & 1.2 & 0.1 & $<0.001$ & 0.80 & 0.73 & $<0.001$ & $<0.001$ \\
\hline Insulin, $\mathrm{nmol} / \mathrm{h}$ & 19 & 23 & 3.1 & 24 & 18 & 2.5 & 0.37 & 0.009 & 0.02 & $<0.001$ & $<0.001$ \\
\hline Glucose, $\mathrm{mmol} / \mathrm{h}$ & 267 & 309 & 21 & 286 & 290 & 18 & 0.29 & 0.93 & 0.50 & $<.0001$ & $<0.001$ \\
\hline Lactate, $\mathrm{mmol} / \mathrm{h}$ & 40 & 42 & 4.8 & 47 & 36 & 3.8 & 0.85 & $<0.001$ & 0.21 & $<.0001$ & 0.19 \\
\hline Urea, mmol/h & -5.6 & -3.0 & 1.7 & -3.5 & -5.1 & 1.3 & 0.30 & 0.25 & 0.40 & 0.28 & 0.64 \\
\hline $\mathrm{NEFA}, \mathrm{mmol} / \mathrm{h}$ & -1.0 & 3.1 & 1.5 & 1.2 & 0.9 & 1.6 & 0.09 & 0.86 & 0.16 & 0.97 & 0.57 \\
\hline $\mathrm{TG}, \mathrm{mmol} / \mathrm{h}$ & -0.1 & -3.5 & 2.7 & -2.8 & -0.8 & 2.8 & 0.44 & 0.64 & 0.85 & 0.51 & 0.60 \\
\hline \multicolumn{12}{|c|}{ Essential AA, mmol/h } \\
\hline Lysine & 5.2 & 5.9 & 0.3 & 5.5 & 5.5 & 0.3 & 0.20 & 0.82 & 0.12 & $<0.001$ & 0.004 \\
\hline Methionine & 1.2 & 1.1 & 0.2 & 1.2 & 1.1 & 0.1 & 0.69 & 0.42 & 0.003 & $<0.001$ & 0.001 \\
\hline Threonine & 3.9 & 4.4 & 0.3 & 4.1 & 4.2 & 0.3 & 0.35 & 0.98 & 0.40 & $<0.001$ & $<0.001$ \\
\hline Tryptophan & 0.9 & 1.0 & 0.1 & 1.0 & 1.0 & 0.1 & 0.27 & 0.78 & 0.81 & $<0.001$ & 0.05 \\
\hline Isoleucine & 5.0 & 5.5 & 0.3 & 5.4 & 5.1 & 0.3 & 0.32 & 0.49 & 0.28 & $<0.001$ & $<0.001$ \\
\hline Leucine & 8.8 & 9.6 & 0.4 & 9.3 & 9.0 & 0.4 & 0.30 & 0.44 & 0.20 & $<0.001$ & $<0.001$ \\
\hline Valine & 6.6 & 7.2 & 0.5 & 6.8 & 7.0 & 0.4 & 0.49 & 0.76 & 0.35 & $<0.001$ & 0.001 \\
\hline Histidine & 2.3 & 2.3 & 0.2 & 2.4 & 2.2 & 0.2 & 0.85 & 0.57 & 0.27 & $<0.001$ & 0.01 \\
\hline Phenylalanine & 5.2 & 5.6 & 0.3 & 5.5 & 5.3 & 0.2 & 0.41 & 0.33 & 0.13 & $<0.001$ & $<0.001$ \\
\hline \multicolumn{12}{|c|}{ Nonessential AA, mmol/h } \\
\hline Alanine & 21.4 & 23.7 & 1.1 & 23.7 & 21.4 & 1.0 & 0.23 & 0.048 & 0.07 & $<0.001$ & $<0.001$ \\
\hline Asparagine & 3.0 & 2.9 & 0.2 & 3.1 & 2.8 & 0.2 & 0.90 & 0.08 & 0.03 & $<0.001$ & $<0.001$ \\
\hline Aspartate & 1.9 & 2.0 & 0.2 & 1.8 & 2.0 & 0.2 & 0.94 & 0.39 & 0.31 & $<0.001$ & 0.48 \\
\hline Cysteine & 2.5 & 3.0 & 0.1 & 2.7 & 2.8 & 0.1 & 0.005 & 0.83 & 0.75 & $<0.001$ & 0.002 \\
\hline Glutamate & -1.5 & -1.2 & 0.3 & -1.5 & -1.1 & 0.3 & 0.53 & 0.28 & 0.37 & 0.02 & 0.79 \\
\hline Glycine & 18.0 & 19.3 & 1.4 & 19.2 & 18.1 & 1.2 & 0.54 & 0.31 & 0.19 & 0.002 & 0.74 \\
\hline Proline & 11.2 & 12.7 & 0.9 & 12.3 & 11.7 & 0.8 & 0.27 & 0.37 & 0.08 & $<0.001$ & 0.03 \\
\hline Serine & 6.8 & 8.0 & 0.5 & 7.5 & 7.2 & 0.4 & 0.16 & 0.33 & 0.17 & $<0.001$ & $<0.001$ \\
\hline Tyrosine & 3.3 & 3.6 & 0.2 & 3.5 & 3.4 & 0.2 & 0.37 & 0.44 & 0.19 & $<0.001$ & $<0.001$ \\
\hline Glutamine & 3.3 & 3.8 & 0.9 & 3.5 & 3.6 & 0.8 & 0.69 & 1.00 & 0.06 & $<0.001$ & 0.15 \\
\hline \multicolumn{12}{|l|}{$\mathrm{VFA}, \mathrm{mmol} / \mathrm{h}$} \\
\hline Acetate & 122.2 & 104.1 & 6.4 & 108.9 & 117.4 & 5.3 & 0.09 & 0.11 & 0.77 & 0.42 & 0.65 \\
\hline Propionate & 56.6 & 47.6 & 5.9 & 52.4 & 51.8 & 4.5 & 0.33 & 0.76 & 0.10 & 0.67 & 0.38 \\
\hline Butyrate & 21.9 & 17.0 & 2.0 & 18.2 & 20.7 & 1.6 & 0.12 & 0.08 & 0.53 & 0.33 & 0.46 \\
\hline Isobutyrate & 1.6 & 1.6 & 0.1 & 1.7 & 1.5 & 0.1 & 0.74 & 0.26 & 0.52 & 0.86 & 0.19 \\
\hline Isovalerate & 1.2 & 1.1 & 0.1 & 1.2 & 1.0 & 0.1 & 0.40 & 0.29 & 0.15 & 0.72 & 0.13 \\
\hline
\end{tabular}

$\mathrm{CON}=$ control $\mathrm{HMB}=\beta$-hydroxy $-\beta$-methyl butyrate; $\mathrm{Trt}=$ treatment; $\mathrm{DIM}=$ days in milk; $\mathrm{ST}=$ sampling time; $\mathrm{NEFA}=$ non-esterified fatty acids; TG = triglycerides; VFA = volatile fatty acid; SEM = standard error of the mean; $\mathrm{AA}=$ amino acid 

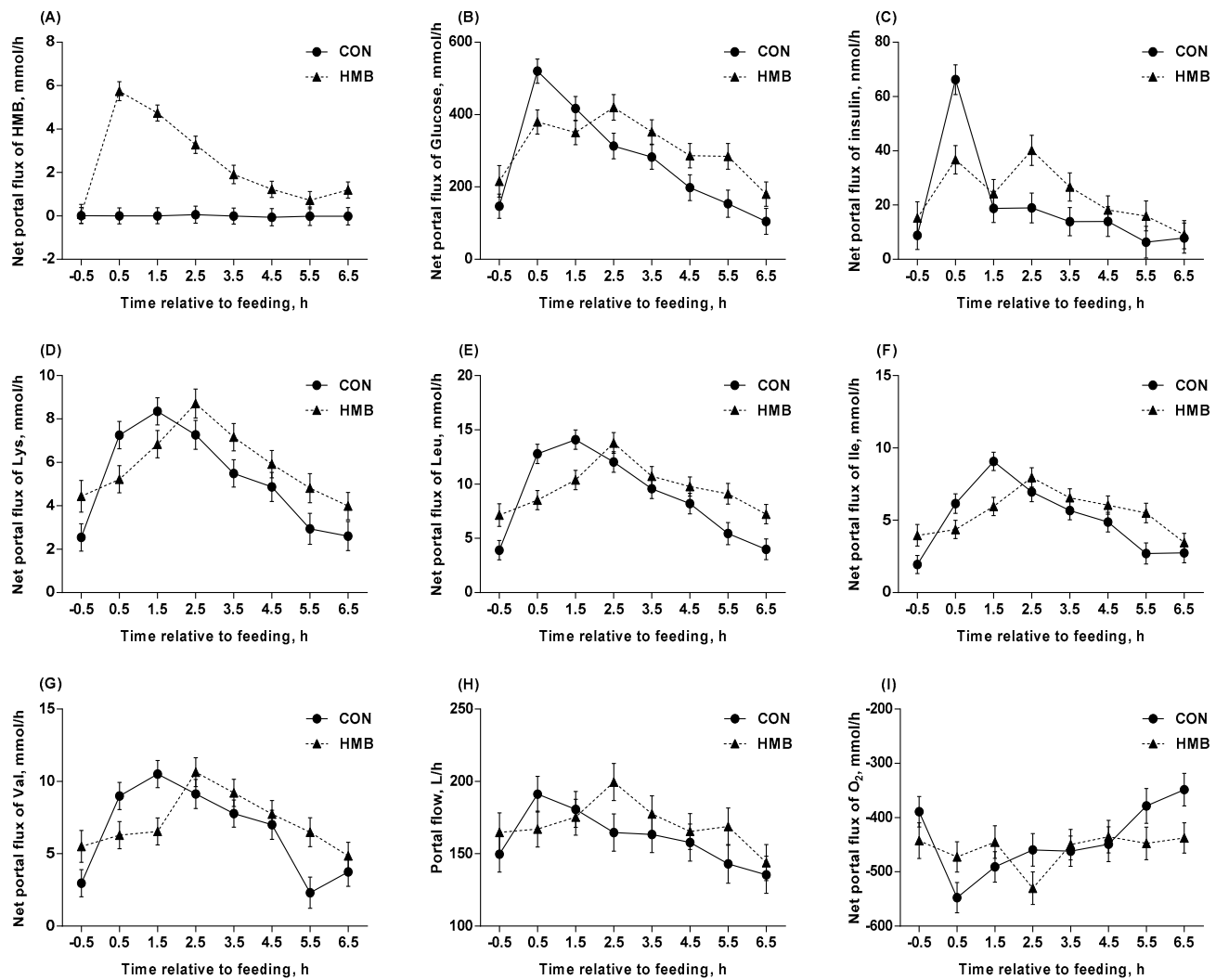

Figure 3. Net portal fluxes of HMB (A), glucose (B), insulin (C), Lys (D), Leu (E), Ile (F), Val (G), portal flow $(\mathbf{H})$, and net portal flux of $\mathrm{O}_{2}(\mathrm{I})$ in control (CON) and HMB-supplemented (HMB) sows during late gestation. Data are expressed as the mean \pm SEM. Sows were regarded as the experimental units, $n=4$ for each group. Interaction of treatment and sampling time after feeding were observed for net portal fluxes of HMB $(p<0.001)$, glucose $(p<0.001)$, insulin $(p<0.001)$, Lys $(p=0.004)$, Leu $(p<0.001)$, Ile $(p<0.001)$, Val $(p=0.001)$, portal flow $(p=0.03)$, and $\mathrm{O}_{2}(p=0.04)$, respectively. $\mathrm{CON}=$ control; $\mathrm{HMB}=\beta$-hydroxy- $\beta$-methyl butyrate.

Table 5. Net portal recovery of amino acids in control (CON) and HMB-supplemented (HMB) sows during late gestation.

\begin{tabular}{|c|c|c|c|c|c|c|c|c|c|}
\hline \multirow{2}{*}{ Item } & \multicolumn{2}{|c|}{ Treatment } & \multirow{2}{*}{ SEM } & \multicolumn{2}{|c|}{ DIM } & \multirow{2}{*}{ SEM } & \multicolumn{3}{|c|}{$p$-Values } \\
\hline & CON & HМB & & -10 & -3 & & Trt & DIM & Trt $\times$ DIM \\
\hline \multicolumn{10}{|l|}{ Essential AA, \% } \\
\hline Lysine & 64.8 & 75.4 & 4.2 & 70.5 & 69.8 & 4.2 & 0.10 & 0.91 & 0.18 \\
\hline Methionine & 49.0 & 45.5 & 6.0 & 48.8 & 45.7 & 6.0 & 0.69 & 0.72 & 0.049 \\
\hline Threonine & 54.3 & 61.5 & 4.6 & 57.4 & 58.4 & 4.6 & 0.28 & 0.88 & 0.47 \\
\hline Tryptophan & 62.9 & 72.0 & 4.1 & 67.8 & 67.1 & 4.1 & 0.14 & 0.90 & 0.67 \\
\hline Isoleucine & 61.9 & 68.5 & 3.5 & 66.8 & 63.6 & 3.5 & 0.21 & 0.53 & 0.29 \\
\hline Leucine & 63.2 & 70.8 & 3.2 & 68.3 & 65.7 & 3.2 & 0.11 & 0.58 & 0.25 \\
\hline Valine & 61.3 & 68.1 & 4.1 & 64.1 & 65.4 & 4.1 & 0.27 & 0.82 & 0.42 \\
\hline Histidine & 57.9 & 58.7 & 4.7 & 62.0 & 54.6 & 4.7 & 0.92 & 0.28 & 0.26 \\
\hline Phenylalanine & 67.8 & 73.7 & 3.5 & 72.1 & 69.3 & 3.5 & 0.26 & 0.59 & 0.23 \\
\hline \multicolumn{10}{|c|}{ Nonessential AA, \% } \\
\hline Alanine & 181.4 & 210.9 & 9.0 & 207.6 & 184.7 & 9.0 & 0.04 & 0.10 & 0.23 \\
\hline Aspartate & 15.5 & 19.8 & 0.8 & 17.7 & 17.6 & 0.8 & 0.003 & 0.97 & 0.96 \\
\hline Cysteine & 51.6 & 53.0 & 5.8 & 49.6 & 55.1 & 5.8 & 0.87 & 0.52 & 0.63 \\
\hline Glutamate & -3.8 & -3.4 & 0.5 & -4.0 & -3.3 & 0.5 & 0.64 & 0.40 & 0.57 \\
\hline Glycine & 126.0 & 140.6 & 8.2 & 137.3 & 129.2 & 8.2 & 0.23 & 0.50 & 0.33 \\
\hline Proline & 65.8 & 77.2 & 5.5 & 73.6 & 69.3 & 5.5 & 0.17 & 0.59 & 0.26 \\
\hline Serine & 58.6 & 69.8 & 4.4 & 65.6 & 62.9 & 4.4 & 0.10 & 0.67 & 0.37 \\
\hline Tyrosine & 86.0 & 95.2 & 4.9 & 92.2 & 89.1 & 4.9 & 0.21 & 0.66 & 0.25 \\
\hline
\end{tabular}

$\mathrm{CON}=$ control; $\mathrm{HMB}=\beta$-hydroxy- $\beta$-methyl butyrate. Trt = treatment; DIM = days in milk; SEM = standard error of the mean; $\mathrm{AA}=$ amino acid. 


\subsection{Net Hepatic Flux}

Sows fed HMB increased the hepatic vein plasma flow $(p=0.002$, Table 6), net hepatic fluxes of $\operatorname{Met}(p<0.001)$, Asn $(p=0.04)$, and $\operatorname{Gln}(p=0.04)$, and tended to increase the hepatic artery plasma flow $(p=0.06)$, net hepatic fluxes of Phe $(p=0.06)$, and Gly $(p=0.07)$. Net hepatic fluxes of HMB, urea, TG, Trp, Ile and Val were more negative at DIM -3 than at DIM -10 $(p<0.05)$. Interaction between treatment and DIM was observed for net hepatic fluxes of HMB $(p=0.02)$ and Met $(p=0.02)$. Net hepatic fluxes of insulin, lactate, urea, Lys, Met, Ile, Leu, Val, Phe, Ala, Asn, Asp, Cys, Glu, Ser and Tyr were affected by ST $(p<0.05)$. Interaction between treatment and ST was observed for net hepatic fluxes of insulin $(p<0.001)$, Thr $(p=0.04)$, Ala $(p=0.03)$, Asp $(p=0.01)$ and Ser $(p=0.002)$.

Table 6. Blood and plasma flow, and net hepatic fluxes of gases and metabolites in control (CON) and HMB-supplemented (HMB) sows during late gestation.

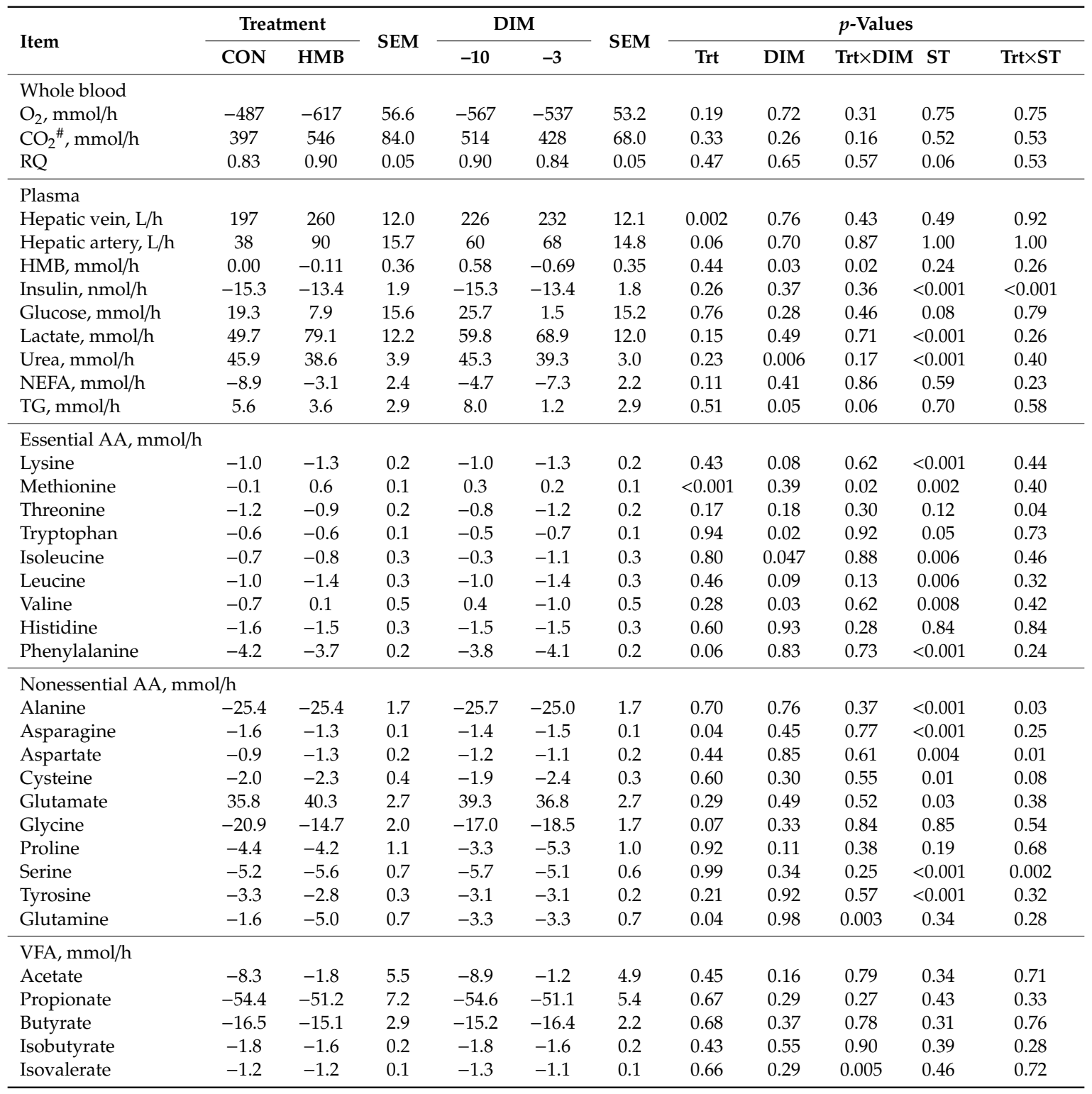

CON = control; HMB = $\beta$-hydroxy- $\beta$-methyl butyrate; Trt = treatment; DIM = days in milk; ST = sampling time; $\mathrm{RQ}=$ respiratory quotient; NEFA = non-esterified fatty acids; $\mathrm{TG}=$ triglycerides; VFA = volatile fatty acid; $\mathrm{SEM}=$ standard error of the mean; $\mathrm{AA}=$ amino acid. ${ }^{\#}$ Corrected for $\mathrm{CO}_{2}$ used for urea synthesis. 


\subsection{Femoral Extraction}

Dietary supplementation of HMB decreased the femoral extractions of Ala and Ser $(p<0.05$; Table 7), and tended to decrease the femoral extractions of insulin $(p=0.09)$ and Glu $(p=0.09)$. Femoral extractions of glucose, Lys, His, Glu, and Gln at DIM -3 were lower than that at DIM $-10(p<0.05)$. Interaction between treatment and DIM was observed for the femoral extraction of propionate $(p<0.05)$. Most of femoral extractions were affected by ST ( $p<0.05)$, except for HMB, urea, NEFA, TG, His, Glu, Gly, Gln, acetate, butyrate and isovalerate. Interaction between treatment and ST was observed for femoral extractions of glucose $(p=0.004)$, lactate $(p=0.006)$, Lys $(p=0.001)$, Ile $(p=0.01)$, Val $(p=0.04)$, Phe $(p=0.01)$, Ala $(p=0.02)$, Cys $(p=0.02)$, and propionate $(p<0.001$; Table 7 and Figure 4$)$.

Table 7. Femoral extraction of metabolites of control (CON) and HMB-supplemented (HMB) sows during late gestation.

\begin{tabular}{|c|c|c|c|c|c|c|c|c|c|c|c|}
\hline \multirow{2}{*}{ Item } & \multicolumn{2}{|c|}{ Treatment } & \multirow{2}{*}{ SEM } & \multicolumn{2}{|c|}{ DIM } & \multirow{2}{*}{ SEM } & \multicolumn{5}{|c|}{$p$-Values } \\
\hline & $\mathrm{CON}$ & НМВ & & -10 & -3 & & Trt & DIM & Trt $\times$ DIM & I ST & $\operatorname{Trt} \times S T$ \\
\hline \multicolumn{12}{|l|}{ Plasma, \% } \\
\hline HMB & 2.9 & 3.0 & 5.1 & 1.8 & 4.1 & 5.4 & 0.93 & 0.72 & 0.50 & 0.16 & 0.63 \\
\hline Insulin & 14.4 & 4.3 & 3.6 & 12.3 & 6.3 & 3.1 & 0.09 & 0.08 & 0.92 & 0.02 & 0.47 \\
\hline Glucose & 9.5 & 8.7 & 0.9 & 10.2 & 8.0 & 0.7 & 0.54 & 0.001 & 0.40 & $<0.001$ & 0.004 \\
\hline Lactate & 26.5 & 24.5 & 2.6 & 24.5 & 26.6 & 2.3 & 0.48 & 0.52 & 0.33 & 0.03 & 0.006 \\
\hline Urea & -0.8 & -0.6 & 0.3 & -0.6 & -0.9 & 0.3 & 0.59 & 0.44 & 0.85 & 0.82 & 0.48 \\
\hline NEFA & 13.0 & -3.2 & 10.1 & 9.5 & 0.3 & 10.0 & 0.19 & 0.39 & 0.71 & 0.14 & 0.72 \\
\hline TG & 0.0 & 1.6 & 2.5 & 0.8 & 0.9 & 2.3 & 0.57 & 0.82 & 0.27 & 0.83 & 0.38 \\
\hline \multicolumn{12}{|l|}{ Essential AA, \% } \\
\hline Lysine & 13.5 & 5.8 & 3.4 & 12.2 & 7.1 & 2.5 & 0.17 & $<0.001$ & 0.30 & $<0.001$ & 0.001 \\
\hline Methionine & 21.1 & 21.7 & 2.3 & 22.0 & 20.9 & 2.3 & 0.72 & 0.82 & 0.22 & 0.09 & 0.90 \\
\hline Threonine & 3.8 & 1.8 & 1.0 & 3.4 & 2.1 & 0.8 & 0.20 & 0.13 & 0.55 & 0.006 & 0.08 \\
\hline Tryptophan & 1.1 & 0.3 & 0.5 & 0.7 & 0.7 & 0.5 & 0.26 & 0.87 & 0.35 & 0.04 & 0.58 \\
\hline Isoleucine & 5.3 & 2.5 & 1.4 & 4.6 & 3.3 & 1.1 & 0.21 & 0.19 & 0.39 & 0.001 & 0.01 \\
\hline Leucine & 5.2 & 2.4 & 1.3 & 4.5 & 3.2 & 1.1 & 0.20 & 0.14 & 0.30 & $<0.001$ & 0.10 \\
\hline Valine & 3.2 & 2.0 & 0.9 & 3.2 & 2.1 & 0.7 & 0.43 & 0.16 & 0.97 & 0.02 & 0.04 \\
\hline Histidine & 2.8 & 1.1 & 1.5 & 4.3 & -0.4 & 1.5 & 0.51 & 0.04 & 0.41 & 0.53 & 0.68 \\
\hline Phenylalanine & 2.9 & 1.3 & 1.2 & 2.6 & 1.6 & 1.0 & 0.48 & 0.23 & 0.39 & $<0.001$ & 0.01 \\
\hline \multicolumn{12}{|c|}{ Nonessential AA, \% } \\
\hline Alanine & 5.9 & 3.2 & 0.7 & 4.5 & 4.5 & 0.6 & 0.04 & 0.77 & 0.55 & $<0.001$ & 0.02 \\
\hline Asparagine & 10.8 & 5.8 & 2.2 & 8.8 & 7.7 & 2.1 & 0.18 & 0.68 & 0.77 & 0.005 & 0.23 \\
\hline Aspartate & 10.4 & 6.5 & 2.9 & 8.6 & 8.3 & 2.3 & 0.38 & 0.79 & 0.65 & 0.04 & 0.33 \\
\hline Cysteine & -1.4 & -0.8 & 0.4 & -0.6 & -1.6 & 0.4 & 0.23 & 0.10 & 0.83 & 0.07 & 0.02 \\
\hline Glutamate & 27.1 & 17.3 & 3.4 & 23.8 & 20.5 & 2.5 & 0.09 & 0.03 & 0.89 & 0.92 & 0.51 \\
\hline Glycine & -0.0 & -0.3 & 0.4 & -0.2 & -0.1 & 0.4 & 0.73 & 0.63 & 0.36 & 0.26 & 0.73 \\
\hline Proline & 1.5 & 1.5 & 0.6 & 1.9 & 1.1 & 0.5 & 0.91 & 0.21 & 0.65 & 0.003 & 0.13 \\
\hline Serine & 12.1 & 8.1 & 1.2 & 10.2 & 9.9 & 1.0 & 0.049 & 0.59 & 0.11 & 0.002 & 0.45 \\
\hline Tyrosine & 1.9 & 1.0 & 1.1 & 1.9 & 1.0 & 0.9 & 0.57 & 0.19 & 0.18 & 0.01 & 0.26 \\
\hline Glutamine & -11.7 & -12.4 & 1.7 & -9.6 & -14.5 & 1.7 & 0.78 & 0.04 & 0.87 & 0.35 & 0.37 \\
\hline \multicolumn{12}{|l|}{ VFA, \% } \\
\hline Acetate & 59.9 & 53.6 & 4.7 & 54.0 & 59.4 & 3.7 & 0.36 & 0.07 & 0.96 & 0.63 & 0.47 \\
\hline Propionate & -830 & 242 & 410 & 281 & -869 & 350 & 0.28 & 0.11 & 0.004 & 0.03 & $<0.001$ \\
\hline Butyrate & 9.0 & 9.4 & 12.1 & 8.7 & 9.7 & 12.1 & 0.85 & 0.81 & 0.33 & 0.09 & 0.57 \\
\hline Isobutyrate & 72.4 & 60.4 & 4.9 & 68.4 & 64.3 & 4.2 & 0.11 & 0.27 & 0.26 & 0.15 & 0.64 \\
\hline Isovalerate & 6.6 & 8.2 & 4.2 & 6.0 & 8.8 & 4.1 & 0.64 & 0.45 & 0.30 & 0.95 & 0.21 \\
\hline
\end{tabular}

$\mathrm{CON}=$ control $\mathrm{HMB}=\beta$-hydroxy- $\beta$-methyl butyrate; Trt = treatment; $\mathrm{DIM}=$ days in milk; $\mathrm{ST}=$ sampling time; NEFA = non-esterified fatty acids; TG = triglycerides; VFA = volatile fatty acid; SEM = standard error of the mean; $\mathrm{AA}=$ amino acid. 

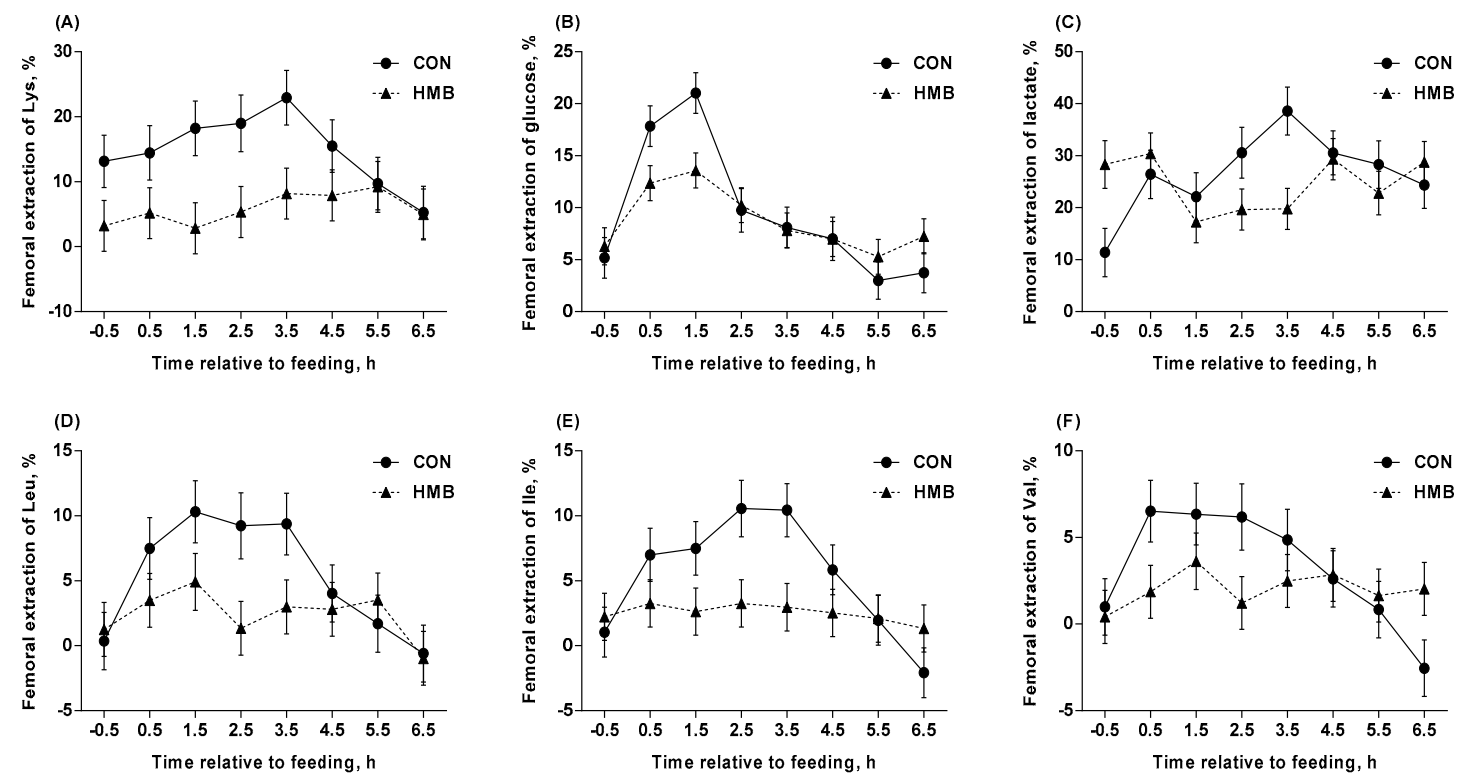

Figure 4. Femoral extractions of Lys (A), glucose (B), lactate (C), Leu (D), Ile (E), and Val (F) in control $(\mathrm{CON})$ and $\mathrm{HMB}$-supplemented (HMB) sows during late gestation. Data are expressed as the mean \pm SEM. Sows were regarded as the experimental units, $n=4$ for each group. Interactions of treatment $(\mathrm{CON}, \mathrm{HMB})$ and sampling time after feeding were observed for femoral extractions of Lys $(p=0.001)$, glucose $(p=0.004)$, lactate $(p=0.006)$, Leu $(p=0.10)$, Ile $(p=0.01)$, and Val $(p=0.04)$, respectively. $\mathrm{CON}=$ control; $\mathrm{HMB}=\beta$-hydroxy- $\beta$-methyl butyrate.

\subsection{HMB Metabolism}

The partitioning of HMB in the HMB supplemented sows revealed that ATTD of HMB was $96 \%$ and $4 \%$ was lost through feces (Figure 5). Furthermore, $8 \%$ of HMB disappeared in the GIT, as evaluated by the difference between the net portal recovery rate $(88 \%)$ and ATTD. The hepatic uptake amounted to $12 \%$ of the HMB intake, whereas, HMB secreted via colostrum and excreted through urine amounted to $26 \%$ and $14 \%$, respectively, relative to intake. Finally, $36 \%$ of HMB relative to intake could not be accounted for quantitatively, but include uptake to muscles, as demonstrated for the hindleg (referred to as "others" in Figure 5).

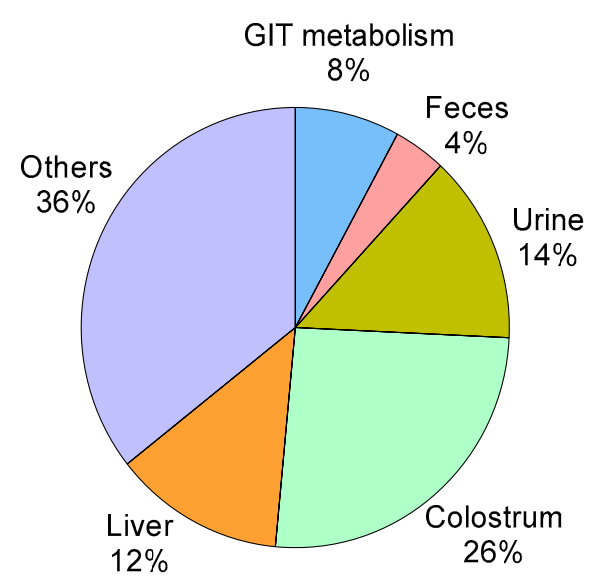

Figure 5. $\beta$-hydroxy- $\beta$-methyl butyrate $(\mathrm{HMB})$ distribution in sow tissues and metabolites (liver, gastrointestinal tract, colostrum, urine, feces, and others) refer to dietary HMB intake. GIT, gastrointestinal tract. The amount of HMB secreted into colostrum is calculated under the assumption that it is taken up by the mammary gland at the day of farrowing [26]. At day -10 and -3 , the $26 \%$ representing colostrum most likely contributes to "Others", as this category is calculated as a difference. 


\section{Discussion}

\subsection{Effects of HMB Supplementation on Reproductive Performance in Late Gestation}

Over the last decades, there has been increased interest in HMB for both animal and human research, due to its nutritional role [27]. The addition of HMB in human has been associated with beneficial health effects for people suffering from several diseases [28]. In animals, numerous studies have shown that dietary supplementation with HMB improved growth and production performance [29]. In the current study, maternal HMB supplementation during the late gestation had no influence on birth weight of piglets, which is in agreement with previous studies [1,5], although other studies have reported increased birth weight in response to maternal HMB supplementation [12,30]. These contradictory results may be due to the varying lengths of HMB administration, meal frequency, the doses of HMB that have been given and not least the number of animals included in the different studies. In a former study, sows fed with $\mathrm{HMB}$ at $2 \mathrm{~g} / \mathrm{d}$ in three equal daily meals for three days prior to parturition increased the fat concentration of sow's colostrum and in turn improved litter growth performance [1]. Flummer and Theil demonstrated that HMB supplemented to sows improved the colostrum production, but inhibited piglet growth at peak lactation [5], which could be due to compromised milk production at peak lactation, due to excessive depletion of labile fat or protein depots around parturition. In the present study, dietary supplementation of HMB increased some AA concentrations (Met, Glu and Pro) in milk, but had no effect on litter performance. This was supported by the milk yield, which did not differ between CON and HMB sows.

\subsection{Effects of $\mathrm{HMB}$ Supplementation on Blood Flow and $\mathrm{O}_{2}$ Consumption}

The portal blood flow may reflect a circulatory response to gastrointestinal function related to the process of digestion and absorption [31]. In the current study, dietary supplementation of HMB increased the portal flow and net portal flux of $\mathrm{O}_{2}$ across sampling time after feeding and suggested that HMB supplementation is beneficial for enhancing transport of absorbed nutrients, and this is supported by improved net portal recovery of AA (some were statistically different, some were numerically greater). Also, dietary supplementation of HMB increased the hematocrit concentration of arterial blood across sampling time after feeding. It is well known that an increased hemoglobin content linked to an increased hematocrit would increase the efficiency of $\mathrm{O}_{2}$ transport [32]. In support of this, HMB supplementation in endurance-trained cyclists has positive effects on aerobic performance by delaying the onset of blood lactate accumulation and extending the time until maximal $\mathrm{O}_{2}$ consumption was reached [33].

The liver plays an essential role in intermediary metabolism and account for a substantial proportion of the whole animal $\mathrm{O}_{2}$ consumption [34]. In this study, dietary supplementation of HMB clearly increased the plasma flow in hepatic vein (197 to $260 \mathrm{~L} / \mathrm{h}, p=0.002)$, which was driven by the increase in hepatic artery plasma flow (38 to $90 \mathrm{~L} / \mathrm{h}, p=0.06$ ), while portal plasma flow was unaffected. The increased hepatic plasma flow resulted in a numerically increased net hepatic uptake of $\mathrm{O}_{2}$. Net hepatic $\mathrm{O}_{2}$ flux were -487 and $-617 \mathrm{mmol} / \mathrm{h}(p=0.19)$ for $\mathrm{CON}$ and $\mathrm{HMB}$ sows, respectively. A negative relationship between blood pressure and plasma flow has been proven in human researches $[35,36]$. Moreover, Nissen et al. have reported that a decrease in systolic blood pressure was observed in humans who were fed $3 \mathrm{~g} \mathrm{HMB} / \mathrm{d}$ [37]. This change in blood pressure may be attributed to the additional calcium intake, due to the HMB supplementation in the form of a calcium salt, and there is experimental evidence showing that calcium supplementation can affect blood pressure [38,39]. Taken together, the increased plasma flow of hepatic vein and in hepatic artery might be ascribed to the decreased blood pressure of sows supplemented with $\mathrm{Ca}(\mathrm{HMB})_{2}$.

\subsection{Effects of HMB Supplementation on Amino Acids Metabolism}

In the current study, dietary supplementation of HMB in sows during late gestation was associated with the increased arterial concentration of Lys and Cys. Results obtained in our study are in accordance 
with the previous investigations on newborn pigs subjected to maternal oral administration of $0.05 \mathrm{~g} / \mathrm{kg}$ of $\mathrm{Ca}(\mathrm{HMB})_{2}$, which reported that plasma concentrations of Glu, Gly, Val, and Tyr increased by 32\%, $30 \%, 23 \%$ and $52 \%$, respectively [30]. Moreover, available data from studies on growing turkeys have shown that 15-week administration with HMB increased concentration of Cys, Glu, Val, Asp, Glu, Pro, Ala, Ile, Leu and Phe, whilst body weights did not differ in comparison to control birds [40]. In studies on pigs subjected to long-term fundectomy (the fundus and glandular part of the cardiac region of the stomach were resected), HMB improved plasma concentration of Met, Thr, Val, Leu, Tyr, Try and Arg, depending on blood sampling time, since last oral dosage of HMB [41]. Taken together, these results indicate an anabolic property of HMB on AA metabolism.

The small intestine of pigs is crucial for the metabolism of AA and nitrogen recycling [42]. Extensive investigations over the past two decades have indicated extensive catabolism of AA by the portal-drained viscera of pigs and humans [32]. The net portal recovery of essential AA are usually stable on $50-70 \%$ after catabolizing by the small intestine in first-pass metabolism [43], which is accordant with our results. Dietary HMB supplementation significantly increased the net portal recovery of some AA (Lys, Ala, Asp and Ser), suggesting that the rate of metabolism of these AA by gastrointestinal tissues (as oxidative fuel) were decreased. In addition, the interaction between treatment and sampling time revealed a more uniform net portal uptake of AA after feeding, and hence, lower maximum absorption peaks for HMB sows as compared with CON sows for all AA except Asp, Glu, Gly, and Gln. These results suggest that HMB supplementation preferably should be done at every single meal to achieve a more constant net absorption rate of nutrients.

The liver plays an important role in the intermediary metabolism of AA; however, there are few reports in the literature describing the net AA metabolism by the liver in nonruminants, particularly in the fed state [44]. In the current study, the hepatic extraction of AA (Met, Thr, Phe, Ala, Asn, Glu, Gly, and Tyr) in HMB supplemented sows were lower than CON sows (data not shown). The decreased hepatic extractions of these AA indicate more AA will be release to peripheral tissues, due to a lower fractional hepatic uptake in the liver. In support of this, we found that the milk AA compositions (Met, Glu and Pro) in HMB supplemented sows were higher than CON sows. Branched chain AA, as the most abundant of essential AA, are not only the substrates for the synthesis of nitrogenous compounds, but they also serve as signaling molecules regulating the metabolism of glucose, lipid, and protein synthesis [45]. It is well known that HMB is responsible partly for the protein anabolic effect of Leu, while neither Ile nor Val have this unique feature, but typically interactions between BCAAs are observed [46]. In the current study, dietary HMB supplementation and sampling time had an interaction on the arterial concentrations, net portal fluxes, and femoral extractions of BCAAs. These results suggested that orally administered HMB could maintain a more stable absorption and metabolism of BCAAs through monitoring arterial concentrations and femoral extractions of BCAAs and delaying the peak of net portal fluxes of BCAAs. In contrast, no impact of HMB or treatment $X$ sampling time interaction was found for BCAAs metabolism in the liver, and support that BCAAs are mainly oxidized peripherally [47].

Numerous studies have suggested $\mathrm{HMB}$ is responsible for inhibiting proteolysis and for modulating protein turnover in vitro and in vivo. Administration with HMB in humans undergoing exercise resulted in increased muscle mass accretion that was associated with inhibition of muscle proteolysis [41]. In the current study, the femoral extractions of Ala, Glu and Ser were markedly decreased by HMB supplementation, which indicated that HMB supplementation could inhibit muscle proteolysis. Similar findings were reported by Nissen et al. who demonstrated that ingestion of $3.0 \mathrm{~g}$ dose of $\mathrm{Ca}(\mathrm{HMB})_{2} / \mathrm{d}$ resulted in decreased release of creatine kinase, an indicator of muscle damage, and 3-methylhistidine, an indicator of muscle protein breakdown [48].

\subsection{Metabolic Fate of $H M B$}

In the current study, HMB was net absorbed and in large quantities shortly after feeding, indicating that this occurs either in the stomach or in the small intestine, because HMB concentration in plasma 
peaked around $30 \mathrm{~min}$ after ingestion. However, the peak of HMB concentration differs from other studies. There may be a number of factors that influence HMB levels in the plasma. In a human study, Vukovich et al. found that $1 \mathrm{~g}$ of HMB-Ca resulted in a peak HMB level in plasma two hours following ingestion, while $3 \mathrm{~g}$ resulted in peak HMB levels $60 \mathrm{~min}$ after ingestion at $300 \%$ greater plasma concentrations ( $487 \mathrm{vs.} 120 \mathrm{nmol} / \mathrm{ml}$ ) [49]. Moreover, comparison of $0.8 \mathrm{~g}$ of HMB-FA (HMB in free acid form) to $1.0 \mathrm{~g}$ HMB-Ca (equivalent amounts of $\mathrm{HMB}$ ) resulted in a doubling of peak plasma levels already after $30 \mathrm{~min}$ in the HMB-FA compared with $120 \mathrm{~min}$ after HMB-Ca consumption [50]. Another study showed that the peak of HMB concentrations was delayed by an hour (from 60 to $120 \mathrm{~min}$ ) when the HMB-Ca dosage was combined with $75 \mathrm{~g}$ of glucose [49]. Taken together, the peak time of appearance of HMB in plasma following ingestion is dependent on the dose, the source of HMB (HMB-FA or HMB-Ca), and whether or not it is consumed with additional nutrients. These findings suggested that microbial fermentation in the stomach or metabolism in the epithelium of the stomach or small intestine is influenced by HMB.

Numerous studies have reported that the effects of oral administration of HMB on health status and growth performance both in human and animals, but the metabolic fate of HMB is not fully understood. In this study, HMB metabolism was studied quantitatively to understand the metabolic fate of HMB by using multiple catheters and an external blood flow marker in a sow model during late gestation. As a result, approximated $88 \%$ (net portal recovery) of HMB intake was net absorbed to the portal vein, and only $4 \%$ of the remaining was lost through feces (96\% ATTD). The gastrointestinal tract, as the main absorption site, can catabolize HMB by the intestinal mucosa during the first pass, as reflected by $8 \%$ of HMB was removed by the GIT in this study. The primary metabolic fate of HMB is its conversion to $\beta$-hydroxy- $\beta$-methylglutaryl-coenzyme $A$, which is a committed step for cholesterol synthesis, but it is unclear what percentage of cholesterol is derived from HMB [2]. In the current study, approximately $12 \%$ of $\mathrm{HMB}$ was catabolized in the liver, and approximately $26 \%$ of $\mathrm{HMB}$ was released into colostrum if it is assumed that HMB transfer to colostrum occurs after parturition starts [26]. Another fate of HMB loss was excretion in the urine, which accounted for $14 \%$. This finding indicates that the kidney does not actively reabsorb HMB. In a former study, approximately $34 \%$ of HMB was lost this way in pigs and sheep [6]. However, a clinical study has shown that only $15 \%$ of a $1 \mathrm{~g}$ dosage of calcium HMB was excreted in the urine, which is consistent with our results [50]. In addition, approximately $36 \%$ of HMB in this study could not be accounted for, because HMB uptake to muscles cannot be measured quantitatively by the multi-catheterized technique. However, the extraction rate of HMB by the femoral muscle support that muscles do indeed metabolize HMB. Metabolism of HMB in skeletal muscle may constitute a greater proportion in late gestation, where HMB is not secreted in colostrum.

\subsection{Effects of HMB Supplementation on Glucogenic, Ketogenic Substrates and Insulin Secretion}

The role of HMB in glucose and insulin homeostasis is controversial [3,51]. Few reports have emerged showing glucose intolerance and elevation in plasma insulin level after HMB administration. In the current study, we found that dietary HMB supplementation decreased the arterial concentrations and net portal fluxes of glucose and insulin after $30 \mathrm{~min}$ of feeding, which suggests that HMB administration improve the insulin sensitivity to a certain extent. In support of this, Sharawy and coworkers reported that dietary supplementation with $\mathrm{HMB}$ might attenuate insulin resistance induced by a high fructose diet in rats through inhibition of glucose transporter-2 in the liver [52]. Recent studies have revealed that $\mathrm{HMB}$ supplementation may alter energy metabolism, as evidenced by increased fat loss during exercise [53]. In fact, HMB has been claimed to stimulate skeletal muscle build-up, while increasing fatty acid oxidation [54]. In this study, dietary supplementation of HMB markedly decreased the arterial concentration of TG. Notably, TG can be stored as lipid droplets within hepatocytes or secreted into the blood, but it also can be hydrolyzed, and the fatty acids may then be metabolized through the $\beta$-oxidation pathway in the liver [55]. The decreased arterial concentration of TG and numerically greater hepatic $\mathrm{O}_{2}$ consumption suggested that HMB supplementation increased fatty 
acid oxidation and the study revealed that this occurred in the muscles and in the liver, although the difference between CON and HMB sows could only be explained by the muscles. Similarly, Rittig et al. used indirect calorimetry to document that HMB administration induced a higher energy expenditure (heat production) and higher lipid oxidation rate [56]. It also increases lipolysis and decreases the content of adipose tissue with no change in body mass, leading to an increase of lipid availability. In addition, $\mathrm{HMB}(0.5 \mu \mathrm{M})$ combined with metformin and resveratrol markedly increases fat oxidation, sirtuin 1 activity, and adenosine monophosphate-activated protein kinase in muscle cells [57].

\section{Conclusions}

This study demonstrated that dietary HMB supplementation resulted in a high and fast net portal absorption of HMB, which in turn increased net portal uptake of amino acids and increased fatty acid oxidation through increasing plasma flow and insulin sensitivity during late gestation. Moreover, the data presented here suggest that $96 \%$ of the HMB ingested was metabolized/excreted/secreted in the body by a number of different organs, including GIT, liver, mammary gland and muscles. These data offer a dynamic metabolic track of orally administered HMB, which could provide essential guidance to improve HMB availability and efficacy to tissues for future research.

Supplementary Materials: The following are available online at http://www.mdpi.com/2072-6643/12/2/561/s1, Table S1: Dietary ingredients and chemical composition of the control diet.

Author Contributions: Conceptualization, P.K.T. and N.B.K.; methodology, P.K.T. and N.B.K.; validation, L.H., P.K.T. and N.B.K.; formal analysis, L.H.; writing-original draft preparation, L.H.; writing-review and editing, L.H., P.K.T. and U.K.; supervision, P.K.T.; project administration, P.K.T.; funding acquisition, P.K.T. All authors have read and agreed to the published version of the manuscript.

Funding: This research was funded by Aarhus University. Liang Hu received a scholarship provided by China Scholarship Council.

Acknowledgments: We wish to thank Birgit Hørdum Løth for the analysis of HMB and Christine Flummer for great help during the practical experiment.

Conflicts of Interest: The authors declare no conflict of interest.

\section{References}

1. Nissen, S.; Faidley, T.D.; Zimmerman, D.R.; Izard, R.; Fisher, C.T. Colostral milk fat percentage and pig performance are enhanced by feeding the leucine metabolite beta-hydroxy-beta-methyl butyrate to sows. J. Anim. Sci. 1994, 72, 2331-2337. [CrossRef]

2. Nissen, S.L.; Abumrad, N.N. Nutritional role of the leucine metabolite $\beta$-hydroxy $\beta$-methylbutyrate (HMB). J. Nutr. Biochem. 1997, 8, 300-311. [CrossRef]

3. Wilson, G.J.; Wilson, J.M.; Manninen, A.H. Effects of beta-hydroxy-beta-methylbutyrate (HMB) on exercise performance and body composition across varying levels of age, sex, and training experience: A review. Nutr. Metab. 2008, 5, 1. [CrossRef]

4. Portal, S.; Eliakim, A.; Nemet, D.; Halevy, O.; Zadik, Z. Effect of HMB supplementation on body composition, fitness, hormonal profile and muscle damage indices. J. Pediatr. Endocrinol. Metab. 2010, 23, 641-650. [CrossRef]

5. Flummer, C.; Theil, P.K. Effect of beta-hydroxy beta-methyl butyrate supplementation of sows in late gestation and lactation on sow production of colostrum and milk and piglet performance. J. Anim. Sci. 2012, 90, 372-374. [CrossRef]

6. Van Koevering, M.; Nissen, S. Oxidation of leucine and alpha-ketoisocaproate to beta-hydroxy-beta-methylbutyrate in vivo. Am. J. Physiol. 1992, 262, E27-E31. [CrossRef]

7. Soumeh, E.A.; van Milgen, J.; Sloth, N.M.; Corrent, E.; Poulsen, H.D.; Nørgaard, J.V. The optimum ratio of standardized ileal digestible isoleucine to lysine for 8-15kg pigs. Anim. Feed Sci. Technol. 2014, 198, 158-165. [CrossRef]

8. Harper, A.E.; Miller, R.H.; Block, K.P. Branched-chain amino acid metabolism. Annu. Rev. Nutr. 1984, 4, 409-454. [CrossRef] 
9. Soumeh, E.A.; Van Milgen, J.; Sloth, N.M.; Corrent, E.; Poulsen, H.D.; Norgaard, J.V. The optimum ratio of standardized ileal digestible leucine to lysine for 8 to $12 \mathrm{~kg}$ female pigs. J. Anim. Sci. 2015, 93, 2218-2224. [CrossRef]

10. Soumeh, E.A.; van Milgen, J.; Sloth, N.M.; Corrent, E.; Poulsen, H.D.; Norgaard, J.V. Requirement of standardized ileal digestible valine to lysine ratio for 8- to 14-kg pigs. Animal 2015, 9, 1312-1318. [CrossRef]

11. Tatara, M.R.; Śliwa, E.; Krupski, W. Prenatal programming of skeletal development in the offspring: Effects of maternal treatment with $\beta$-hydroxy- $\beta$-methylbutyrate (HMB) on femur properties in pigs at slaughter age. Bone 2007, 40, 1615-1622. [CrossRef]

12. Wan, H.F.; Zhu, J.T.; Shen, Y.; Xiang, X.; Yin, H.J.; Fang, Z.F.; Che, L.Q.; Lin, Y.; Xu, S.Y.; Feng, B.; et al. Effects of Dietary Supplementation of beta-hydroxy-beta-methylbutyrate on Sow Performance and mRNA Expression of Myogenic Markers in Skeletal Muscle of Neonatal Piglets. Reprod. Domest. Anim. 2016, 51, 135-142. [CrossRef]

13. Sangild, P.T. Gut responses to enteral nutrition in preterm infants and animals. Exp. Biol. Med. 2006, 231, 1695-1711. [CrossRef]

14. Kristensen, N.B.; Norgaard, J.V.; Wamberg, S.; Engbaek, M.; Fernandez, J.A.; Zacho, H.D.; Poulsen, H.D. Absorption and metabolism of benzoic acid in growing pigs. J. Anim. Sci. 2009, 87, 2815-2822. [CrossRef]

15. Flummer, C.; Lyby, H.; Storli, K.S.; Bjerre-Harpoth, V.; Nielsen, B.M.; Kramer, M.; Rojen, B.A.; Kristensen, N.B.; Theil, P.K. Effects of beta-hydroxy beta-methyl butyrate supplementation to sows in late gestation on absorption and hepatic metabolism of glucose and amino acids during transition. J. Anim. Sci. 2012, 90, 146-148. [CrossRef]

16. Theil, P.K.; Lauridsen, C.; Quesnel, H. Neonatal piglet survival: Impact of sow nutrition around parturition on fetal glycogen deposition and production and composition of colostrum and transient milk. Animal 2014, 8, 1021-1030. [CrossRef]

17. Stoldt, W. Proposal for simplifying fat determination in food. Fats Soaps 1952, 54, $206-207$.

18. Harvey, R.B.; Brothers, A.J. Renal Extraction of Para-Aminohippurate and Creatinine Measured by Continuous in Vivo Sampling of Arterial and Renal-Vein Blood. Ann. N. Y. Acad. Sci. 1962, 102, 46-54. [CrossRef]

19. Marsh, W.H.; Fingerhut, B.; Miller, H. Automated and Manual Direct Methods for the Determination of Blood Urea. Clin. Chem. 1965, 11, 624-627. [CrossRef]

20. Kristensen, N.B. Quantification of whole blood short-chain fatty acids by gas chromatographic determination of plasma 2-chloroethyl derivatives and correction for dilution space in erythrocytes. Acta Agric. Scand. A-Anim. Sci. 2000, 50, 231-236. [CrossRef]

21. Krogh, U.; Oksbjerg, N.; Storm, A.C.; Feyera, T.; Theil, P.K. Mammary nutrient uptake in multiparous sows fed supplementary arginine during gestation and lactation. J. Anim. Sci. 2017, 95, 2517-2532. [CrossRef]

22. Theil, P.K.; Nielsen, T.T.; Kristensen, N.B.; Labouriau, R.; Danielsen, V.; Lauridsen, C.; Jakobsen, K.J.A.A.S. Section A-Animal Science. Estimation of milk production in lactating sows by determination of deuterated water turnover in three piglets per litter. Acta Agric. Scand. A-Anim. Sci. 2002, 52, 221-232.

23. Stein, H.H.; Seve, B.; Fuller, M.F.; Moughan, P.J.; de Lange, C.F.M. Invited review: Amino acid bioavailability and digestibility in pig feed ingredients: Terminology and application. J. Anim. Sci. 2007, 85, 172-180. [CrossRef]

24. Hu, L.; Kristensen, N.B.; Che, L.; Wu, D.; Theil, P.K. Net absorption and liver metabolism of amino acids and heat production of portal-drained viscera and liver in multiparous sows during transition and lactation. J. Anim. Sci. Biotechnol. 2020, 11. [CrossRef]

25. Feyera, T.; Zhou, P.; Pedersen, T.F.; Larsen, U.K.; Theil, P.K. Comparison of urine production measured by total collection and para-aminohippuric acid infusion methods. In Proceedings of the 14th International Symposium on Digestive Physiology of Pigs (DPP2018), Brisbane, Australia, 21-24 August 2018.

26. Feyera, T.; Zhou, P.; Nuntapaitoon, M.; Sorensen, K.U.; Krogh, U.; Bruun, T.S.; Purup, S.; Jorgensen, H.; Poulsen, H.D.; Theil, P.K. Mammary metabolism and colostrogenesis in sows during late gestation and the colostral period. J. Anim. Sci. 2019, 97, 231-245. [CrossRef]

27. Molfino, A.; Gioia, G.; Rossi Fanelli, F.; Muscaritoli, M. Beta-hydroxy-beta-methylbutyrate supplementation in health and disease: A systematic review of randomized trials. Amino Acids 2013, 45, 1273-1292. [CrossRef]

28. Holecek, M. Beta-hydroxy-beta-methylbutyrate supplementation and skeletal muscle in healthy and muscle-wasting conditions. J. Cachexia Sarcopenia Muscle 2017, 8, 529-541. [CrossRef] 
29. Szczesniak, K.A.; Ostaszewski, P.; Fuller, J.C., Jr.; Ciecierska, A.; Sadkowski, T. Dietary supplementation of beta-hydroxy-beta-methylbutyrate in animals-A review. J. Anim. Physiol. Anim. Nutr. 2015, 99, 405-417. [CrossRef]

30. Tatara, M.R.; Krupski, W.; Tymczyna, B.; Studzinski, T. Effects of combined maternal administration with alpha-ketoglutarate (AKG) and beta-hydroxy-beta-methylbutyrate (HMB) on prenatal programming of skeletal properties in the offspring. Nutr. Metab. 2012, 9, 39. [CrossRef]

31. Gonzalez-Valero, L.; Rodriguez-Lopez, J.; Lachica, M.; Fernandez-Figares, I. Differences in portal appearance of lysine and methionine in Iberian and Landrace pigs. J. Anim. Sci. 2012, 90, 110-112. [CrossRef]

32. Tan, B.; Li, X.; Wu, G.; Kong, X.; Liu, Z.; Li, T.; Yin, Y. Dynamic changes in blood flow and oxygen consumption in the portal-drained viscera of growing pigs receiving acute administration of (L)-arginine. Amino Acids 2012, 43, 2481-2489. [CrossRef]

33. Vukovich, M.D.; Dreifort, G.D. Effect of beta-hydroxy beta-methylbutyrate on the onset of blood lactate accumulation and V (O)(2) peak in endurance-trained cyclists. J. Strength Cond. Res. 2001, 15, 491-497.

34. Simoes Nunes, C.; Galibois, I.; Rerat, A.; Savoie, L.; Vaugelade, P. Hepatic and portal-drained viscera balances of amino acids, insulin, glucagon and gastrin in the pig after ingestion of casein or rapeseed proteins. Reprod. Nutr. Dev. 1991, 31, 217-231. [CrossRef]

35. Groves, A.M.; Kuschel, C.A.; Knight, D.B.; Skinner, J.R. Relationship between blood pressure and blood flow in newborn preterm infants. Arch. Dis. Child. Fetal Neonatal Ed. 2008, 93, F29-F32. [CrossRef]

36. Schouten, W.R.; Briel, J.W.; Auwerda, J.J.A. Relationship between Anal Pressure and Anodermal Blood-Flow-The Vascular Pathogenesis of Anal Fissures. Dis. Colon Rectum 1994, 37, 664-669. [CrossRef]

37. Nissen, S.; Sharp, R.L.; Panton, L.; Vukovich, M.; Trappe, S.; Fuller, J.C. beta-hydroxy-beta-methylbutyrate (HMB) supplementation in humans is safe and may decrease cardiovascular risk factors. J. Nutr. 2000, 130, 1937-1945. [CrossRef]

38. McCarron, D.A.; Reusser, M.E. Finding consensus in the dietary calcium-blood pressure debate. J. Am. Coll. Nutr. 1999, 18, 398S-405S. [CrossRef]

39. Birkett, N.J. Comments on a meta-analysis of the relation between dietary calcium intake and blood pressure. Am. J. Epidemiol. 1998, 148, 223-228. [CrossRef]

40. Tatara, M.R. Effect of beta-hydroxy-beta-methylbutyrate (HMB) administration on volumetric bone mineral density, and morphometric and mechanical properties of tibia in male turkeys. J. Anim. Physiol. Anim. Nutr. 2009, 93, 669-677. [CrossRef]

41. Tatara, M.R.; Sliwa, E.; Krupski, W.; Worzakowska, M. 3-Hydroxy-3-methylbutyrate administration diminishes fundectomy-induced osteopenia of the lumbar spine in pigs. Nutrition 2008, 24, 753-760. [CrossRef]

42. Stoll, B.; Henry, J.; Reeds, P.J.; Yu, H.; Jahoor, F.; Burrin, D.G. Catabolism dominates the first-pass intestinal metabolism of dietary essential amino acids in milk protein-fed piglets. J. Nutr. 1998, 128, 606-614. [CrossRef]

43. Wu, G.; Bazer, F.W.; Davis, T.A.; Kim, S.W.; Li, P.; Marc Rhoads, J.; Carey Satterfield, M.; Smith, S.B.; Spencer, T.E.; Yin, Y. Arginine metabolism and nutrition in growth, health and disease. Amino Acids 2009, 37, 153-168. [CrossRef]

44. Stoll, B.; Burrin, D.G. Measuring splanchnic amino acid metabolism in vivo using stable isotopic tracers. J. Anim. Sci. 2006, 84, E60-E72. [CrossRef]

45. Nie, C.; He, T.; Zhang, W.; Zhang, G.; Ma, X. Branched Chain Amino Acids: Beyond Nutrition Metabolism. Int. J. Mol. Sci. 2018, 19, 954. [CrossRef]

46. Holecek, M.; Muthny, T.; Kovarik, M.; Sispera, L. Effect of beta-hydroxy-beta-methylbutyrate (HMB) on protein metabolism in whole body and in selected tissues. Food Chem. Toxicol. 2009, 47, 255-259. [CrossRef]

47. Kristensen, N.B.; Wu, G. Metabolic functions of the porcine liver. In Nutritional Physiology of Pigs; SEGES: Copenhagen, Denmark, 2012; pp. 1-17. Available online: https://svineproduktion.dk/Services/ Undervisningsmateriale2 (accessed on 20 February 2020).

48. Nissen, S.; Sharp, R.; Ray, M.; Rathmacher, J.; Rice, D.; Fuller, J., Jr.; Connelly, A.; Abumrad, N.J.J.O.A.P. Effect of leucine metabolite $\beta$-hydroxy- $\beta$-methylbutyrate on muscle metabolism during resistance-exercise training. J. Appl. Physiol. 1996, 81, 2095-2104. [CrossRef]

49. Vukovich, M.D.; Slater, G.; Macchi, M.B.; Turner, M.J.; Fallon, K.; Boston, T.; Rathmacher, J. beta-hydroxy-beta-methylbutyrate (HMB) kinetics and the influence of glucose ingestion in humans. J. Nutr. Biochem. 2001, 12, 631-639. [CrossRef] 
50. Fuller, J.C., Jr.; Sharp, R.L.; Angus, H.F.; Baier, S.M.; Rathmacher, J.A. Free acid gel form of beta-hydroxy-beta-methylbutyrate (HMB) improves HMB clearance from plasma in human subjects compared with the calcium HMB salt. Br. J. Nutr. 2011, 105, 367-372. [CrossRef]

51. Yonamine, C.Y.; Teixeira, S.S.; Campello, R.S.; Gerlinger-Romero, F.; Rodrigues, C.F., Jr.; Guimaraes-Ferreira, L.; Machado, U.F.; Nunes, M.T. Beta hydroxy beta methylbutyrate supplementation impairs peripheral insulin sensitivity in healthy sedentary Wistar rats. Acta Physiol. 2014, 212, 62-74. [CrossRef]

52. Sharawy, M.H.; El-Awady, M.S.; Megahed, N.; Gameil, N.M.J.C.J.O.P. The ergogenic supplement $\beta$-hydroxy- $\beta$-methylbutyrate (HMB) attenuates insulin resistance through suppressing GLUT-2 in rat liver. Can. J. Physiol. Pharm. 2015, 94, 488-497. [CrossRef]

53. Bruckbauer, A.; Zemel, M.B.; Thorpe, T.; Akula, M.R.; Stuckey, A.C.; Osborne, D.; Martin, E.B.; Kennel, S.; Wall, J.S. Synergistic effects of leucine and resveratrol on insulin sensitivity and fat metabolism in adipocytes and mice. Nutr. Metab. 2012, 9, 77. [CrossRef]

54. Cheng, W.; Phillips, B.; Abumrad, N. Beta-hydroxy-beta-methyl butyrate increases fatty acid oxidation by muscle cells. FASEB J. 1997, 11, 2204.

55. Cottrell, E.C.; Ozanne, S.E. Developmental programming of energy balance and the metabolic syndrome. Proc. Nutr. Soc. 2007, 66, 198-206. [CrossRef]

56. Rittig, N.; Bach, E.; Thomsen, H.H.; Moller, A.B.; Hansen, J.; Johannsen, M.; Jensen, E.; Serena, A.; Jorgensen, J.O.; Richelsen, B.; et al. Anabolic effects of leucine-rich whey protein, carbohydrate, and soy protein with and without beta-hydroxy-beta-methylbutyrate (HMB) during fasting-induced catabolism: A human randomized crossover trial. Clin. Nutr. 2017, 36, 697-705. [CrossRef]

57. Bruckbauer, A.; Zemel, M.B. Synergistic effects of metformin, resveratrol, and hydroxymethylbutyrate on insulin sensitivity. Diabetes Metab. Syndr. Obes. 2013, 6, 93-102. [CrossRef]

(C) 2020 by the authors. Licensee MDPI, Basel, Switzerland. This article is an open access article distributed under the terms and conditions of the Creative Commons Attribution (CC BY) license (http://creativecommons.org/licenses/by/4.0/). 